LA- $-9405-S R$

DE82 019718

\title{
The Evolution of LAMPF and Its Research Programs
}

\author{
Louis Rosen
}
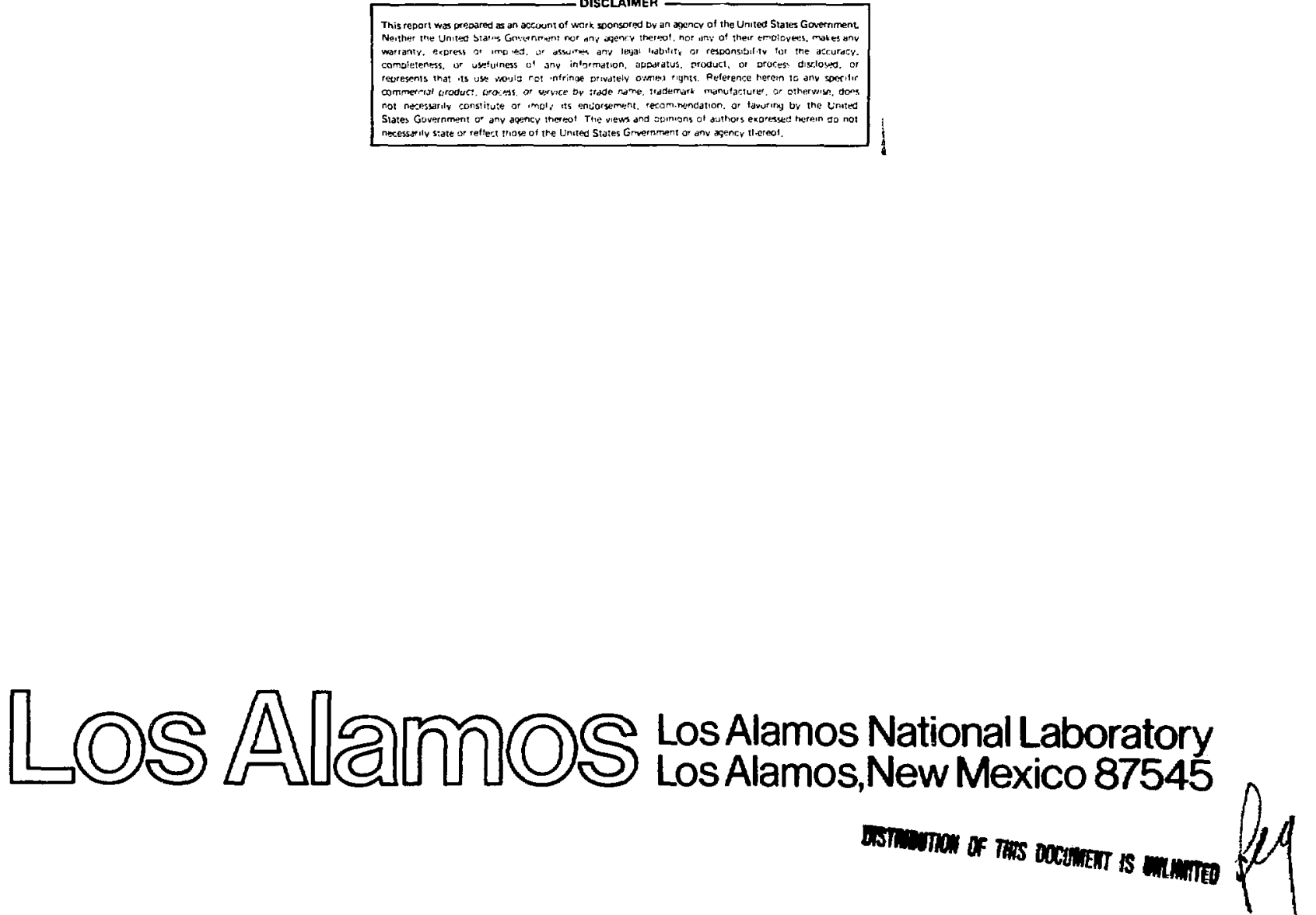
THE EVOLUTION OF LAMPF AND ITS RESEARCH PROGRAMS

by

Louis Rosen

August 12, 1981

\begin{abstract}
The multiple beam 7 ines and the variety of particle beams at the Clinton P. Anderson Meson Physics Facility (LAMPF) permit a wide range of experiments in particle and nuclear physics and nuclear chemistry. Some of the principal achievements at LAMPF during the past few years are described.
\end{abstract}

\title{
I. INTRODUCTION
}

It appears that the more we depend on science and technology for military and economic security, physical well-being, and international leadership, the more we are obliged to articulate and defend the role of science in our society.

In a talk to Episcopal Deans not long ago, I pointed out that one can make an interesting analogy between religion and science. Both are based on faith and require sacrifice. Both hold out promises for the future, al though science may provide more options, assuming that good science is analogous to heaven and bad science is analogous to hell. Neither can make guaranteed promises. Both science and religion strive for harmony with cosmic forces and the laws by which they are governed. Then I made the mistake of pointing out that essentially all sources of energy, on earth and in the heavens above, are nuclear in origin. However, on that fact rests the ultimate justification for LAMPF.

If we divide the study of the fundamental properties of matter into subnuclear science and nuclear science, LAMPF is presently the largest nuclear-science facility in the world (Fig. 1). The LAMPF real-life reason for being is shown, simplified, in Fig. 2. The content of the Universe may be divided into matter, particles (baryons and leptons) which have half-integer spin and obey the Pauli exclusion principle, and interactions, which are represented by integer-spin 
fields and which do not obey the exclusion principle. The interactions comprise four forces: the strong forces interact only with hadrons; the electromagnetic forces with charged hadrons and leptons; the weak forces interact with hadrons and leptons; finally, the gravitationa? forces interact with everything. LAMPF provides the probes with which to explore forces and interactions. However, LAMPF is used for much more, as we shall see. I shall talk about LAMPF goals and objectives and overall programs, and I shall show examples of the kinds of data we are obtaining. I shall also indicate something about our future directions. Finally, I want to tell you a little about our applied research.

\section{EVOLUTION OF LAMPF}

But first let's have a microlook at the evolution of LAMPF. Figures 3 to 9 describe the evolution of LAMPF. I call it a microlook because LAMPF has been in evolution for about $10^{7}$ minutes, and 1 will discuss that evolution in about 10 minutes.

\section{PURPOSES OF LAMPF}

Figure 10 summarizes our hopes for LAMPF in a very general way. These were our original goals and objectives, and they remain valid today. In fact, every activity indicated is presently under way.

The purpose of nuclear physics has always been to understand atomic nuclei-their structure, their properties, and their interactions--in terms of basic constituents and basic forces. It appears that it is now left mainly to mediumenergy physics to complete this goal, while it also provides a bridge between nuclear and subnuclear physics. If, indeed, strong interactions are best understood in terms of colored quarks and vector bosons (gluons), then the particles detected in nuclear physics are all many-body objects, and the study of mesons and nucleons in terms of quarks and gluons becomes, for the nuclear physicist, a not unfamiliar many-body problem.

LAMPF is programmatically oriented. Most of our activities are "bread-andbutter" ones. Figures 11 to 14 identify the kinds of programs presently under way. The forces between nucleons remain a basic challenge. The long-range forces (1-3 fm) appear to be describable in terms of single- and two-pion exchanges. The short-range force presents a problem. How is it affected by the quark structure of nucleons and by the delta within the nucleus?

Only 20\% of all unstable nuclei have been explored. More are being identified. However, we do not yet understand even the simple modes of motion 
with in nuclear matter. The giant monopole resonance is an especially interesting object. It entails radial vibrations; the neutrons and protons can be in phase (isoscalar) or out of phase (isovector). The nucleus breathes, thus bearing on the nuclear-density property. This means that one may be able to derive from such studies information on the compressibility of nuclear matter, which has great astrophysical significance. One may hope to distinguish a mode of motion where the neutrons and protons together perform radial oscillations, from one where the neutrons move in one radial direction, while the protons are $180^{\circ}$ out of phase. The giant isovector dipole resonance was the first collective mode discovered. It is excited by photons or electrons and involves coherent vibration of protons out of phase with neutrons. Alpha particles were used to discover the isoscalar monopole giant resonance, in which surface nucleons move against the core. The isovector monopole mode is excited by the $\left(\pi^{-}, \pi^{0}\right)$ reaction, causing surface neutrons to oscillate against protons. This is an example of the complementarities of nucleon probes.

The fact that nuclear reactions usually proceed through intermediate stages, from direct to many-body interactions, has a bearing on the general many-body problem in physics.

The measurement and understanding of charge and matter distributions in nuclei remain a primary goal in nuclear science. It would appear that many different probes will be required for unambiguous resolution of this problem.

LAMPF is applicable to experiments that require high flux and/or high precision.

It is important to find the 1 imits of quantum electrodynamics (QED), because these may yet provide the proper framework for understanding all physical phenomena. Figure 15 indicates the kinds of questions we are asking in this domain. IV. ATOMIC PHYSICS

A number of experiments are under way in atomic physics at electron-volt energies.

\section{A. $H^{-}$Photodetachment Studies (Colliding-Beam Experiments)}

Colliding beams are in vogue. We do it with mirrors. For photodissociation of $\mathrm{H}^{-}$we employ colliding beams, as illustrated in Fig. 16, to achieve center-ofmass energies of 0.5-20 eV with milli-electron-volt resolution. There are no tunable vacuum ultraviolet lasers, but the Doppler effect permits the use of a green laser to study photodissociation resonances with exquisite resolution. A number of resonances have been resolved for the first time. 
These experiments test our understanding of scattering theory in a system with well-known dynamics. The two-electron detachment process represents the long-range three-body problem. There is a dramatic strong-field Stark effect discovered in the Feshbach and shape resonances; the fumer exhibit splitting into three resonances at low fields and completely disappear at higher fields. The shape resonance is almost unaffected by an eiectric field except for some broadening at $1 \mathrm{MV} / \mathrm{cm}$. A pulsed magnet, yielding an equivalent electric field $(v \times B)$ of $7-10 \mathrm{MV} / \mathrm{cm}$, will be used in future experiments. Figures 17 and 18 show some results.

B. Muonium

Muonium is a light isotope of hydrogen with similar energy levels. Although $\Delta v_{H}$ has been measured to one part in $10^{12}$, this is not a good test of QED because $\Delta v_{H}$ can only be calculated to $3 \mathrm{ppm}$ due to lack of knowledge about polarizability of the proton. Muonium, on the other hand, is composed of leptons that can be treated as structureless point particles. To produce muonium requires muons. The kinds of muon beams obtained at LAMPF are shown in Fig. 19.

The hyperfine structure of muonium has been measured, as has also the ratio of the muon to proton magnetic moment. One uses microwave magnetic resonance techniques, Fig. 20. $\mu^{+}$are stopped in a resonant cavity containing $\sim 0.5 \mathrm{~atm}$ $\mathrm{Kr}$. The four Zeeman levels in the metastable slot are split by a 14-kG magnetic field. Transitions are induced by pulsing the microwave frequency between the two transition frequencies. The sum and difference of the two transition frequencies yield the muonium hfs interval and the muon magnetic moment. The measured value of the $\mu_{u} / \mu_{p}$ also yields the ratio of the muon mass to that of the proton. Such measurements provide a test of the validity of the theory of boundstate QED. Alternatively, if you assume correctness of QED, the experimental values of $\Delta v$ and $\mu_{u} / \mu_{p}$ provide a value of $\alpha$, the fine structure constant. Because of parity nonconservation, the $\mu^{+}$obtainea from decay of $\pi^{+}$are polarized. This in turn means that their decay positrons are emitted preferentially in the direction opposite to the $\mu^{+}$momentum. It is therefore polarized $\mu^{+}$that are stopped in krypton gas inside the resonant microwave cavity surrounded by a magnetic field, the axis of which is parallel to the muon spin. Muonium is formed in two states because of the muon polarization and the strong longitudinal field. Microwave-induced transitions are revealed by the decay positrons, which are ejected mainly in the direction of muon spin, because the 
transitions flip the spin of the muon in the direction opposite to the initial beam polarization. If, therefore, one positions a telescope in the direction opposite to the initial beam polarization, there will be a strong enhancement of positron counts with respect to the microwaves-off condition. In normal $\pi$-decay, the backward-decaying muons are polarized in the forward direction. However, since the surface muon beam is polarized in the direction opposite to its momentum, positrons from muons that decay without forming muonium are predominantly ejected away from the positron telescope. High intensity i,s necessary because observation must be extrapolated to zero pressure. A surface muon beam is used.

Muonium is a superb system for study of the electromagnetic interaction of two different leptons.

1. LAMPF is an excellent source of stopped muons. We can obtain an average stopping rate of $10^{6} / \mathrm{s}^{+}$in a very thin target $(0.5-\mathrm{atm}$ krypton gas, $20 \mathrm{~cm}$ in length).

2. Figure 21 shows the spectroscopy of muonium. The his interval $\Delta v$ has been measured to $0.6 \mathrm{ppm}$ and $\mu_{\mu} / \mu_{\mathrm{p}}$ to $0.8 \mathrm{ppm}$. This is a classic muonium microwave magnetic-resonance experiment, which measures the Zeeman effect.

3. We have observed fast muonium in vacuum, which will permit a search for Lyman $\alpha$-photons from quenched metastable 2s muonium. Measurements will be made of the muonium Lamb shift and also of muonium-antimuonium conversion. Muonium in vacuum is a clean fundamental system. The Lamb shift (and fine structure splitting) of $n=2$ states can be measured.

C. Dalitz Decay of Neutral Pions - Another Test of QED

The decay rate for $\pi^{0} \rightarrow e^{+} e^{-} \gamma$ relative to $\pi^{0} \rightarrow \gamma$ is calculable using conventional QED.

The $\pi^{0}$ is produced from $\pi^{-} p+\pi^{0} n$, with measured ratios $(1.25 \pm 0.04) \times 10^{-2}$. The ratio

$$
\frac{\Gamma\left(\pi^{0}+e^{+} e^{-} \gamma\right)}{\Gamma\left(\pi^{0}+2 \gamma\right)}
$$

is quite sensitive to vacuum polarization contributions.

\section{NUCLEON-NUCLEON INTERACTIONS}

The two-nucleon problem (Fig. 22) is of interest in its own right and also because, without its solution, we are stymied in our attempts to use protons to explore the nucleus. 
One major aim of this program is to measure the scattering amplitudes as a function of energy for the nucleon-nucleon system. These amplitudes can then be systematized by making energy-dependent partial-wave decompositions. Such phaseshift analysis permits use of the data to understand nucleon-nucleus interaction and derive nuclear-structure data from them.

Polarized beams and polarized targets are in use to elucidate all the spindependent parts of the scattering amplitude. To do this it is essential to measure cross sections in pure spin states. We see the isovector nucleon-nucleon amplitudes. At least nine independent experiments must be performed--cross sections, analyzing power, spin correlation parameters, and spin depolarization and transfer parameters.

The $p-p$ total cross sections for beam and target protons in pure parallel and antiparallel longitudinal spin states for energies between 300 and $800 \mathrm{MeV}$ have been measured.

Polarized Target: $\mathrm{C}_{3} \mathrm{H}_{8} \mathrm{O}_{2}$ (Propanediol)

The polarization of beam and target can be $80 \%$ each. One purpose is to explore for dibaryon resonances and to determine whether such might be due to quark states.

Parity-violation (PV) experiments are under way and will tell us the extent to which there is interference between the strong and weak forces. These are predicted to produce PV at the level of $10^{-7}$. Measurements at low energies yield a PV asymmetry $\sim 10^{-7}$, in agreement with calculations.

A more recent motivation for nucleon-nucleon scattering is the detection of dibaryon resonances. These are predicted by calculation of the angular-momentum excitation of six quarks confined in a bag, so one might conceivabiy learn about the mechanism of quark confinernent from intermediate-energy NN studies. Figure 23 shows some recent data.

Cross sections and asymmetries depend mainly on central and spin-orbit parts of the force. However, triple-scattering experiments are sensitive to spin-spin interaction terms, which turn out to be very important. They are relevant to identifying dibaryon resonances.

VI. TESTS OF CHARGE SYMMETRY

A. Forward-Angle Quas i-Free Proton-Neutron Analyzing Powers

800-MeV Polarized Protons Scattered from Liquid Deuterium (Impulse Approximation). 
Data show reasonably good agreement of quasi-free results with free nucleonnucleon scattering. This is expected because the final-state interaction should be weak at the energies involved. We are attempting to detect small violations of conservation and symetry 1aws. We pay heed to Einstein's dictum: "Symmetry dictates the dynamics of nature."

A substantial effort to detect charge-symmetry violation has been undertaken. Charge independence, which implies that $\mathrm{pp}, \mathrm{np}$, and $\mathrm{nn}$ forces are equal to each other in the same space-spin states, is not strictly valid, presumably because of the mass difference of neutral and charged mesons exchanged between nucleons. However, no violation of charge symmetry has been unambiguously detected. But suspicions have been raised from comparison of binding energies of mirror nuclei $\left({ }^{3} \mathrm{He}-{ }^{3} \mathrm{H}\right)$ and an apparent inequality of the nuclear part of the $\pi^{ \pm}$-deuterium scattering cross section observed some years ago.

Charge symmetry requires that a charge-reflected system behave in identical fashion to the original one, implies the equality of $\mathrm{nn}$ and pp nuclear forces, and has implications for the np system.

We are testing charge symmetry in a number of ways, e.g., $p+d$ and $n+d$. Comparison of $n-n$ and $p-p$ scattering involves large coulomb corrections (factor of 2) and nuclear-structure corrections, since nn scattering must be deduced from $n-D$ scattering.

A test of charge symmetry in the $n p \rightarrow d \pi^{0}$ reaction has been carried out at $795 \mathrm{MeV}$.

The angular distribution of $n p \rightarrow d \pi^{\circ}$ is symetric about $90^{\circ}$ to within $\pm 0.5 \%$ and is consistent with charge symmetry to better precision than previously obtained. Figure 24 shows recent results. Another test of charge-symmetrybreaking forces is obtained by comparing high-precision measurements of the zerocrossing angle $\left(\theta_{0}\right)$ for the analyzing power in $\overrightarrow{n p}$ and $\overrightarrow{n p}$ scattering, when $\theta_{0}$ is plotted vs energy. This tests the forces that cause isospin mixing in the twobody system (Fig. 25).

\section{B. $\pi^{ \pm} D$ Scattering}

The scattering of $\pi^{ \pm}$by deuterium provides a direct test of charge symmetry, while minimizing Coulomb and nuclear-structure corrections.

The $\pi^{ \pm}$-deuterium scattering experiment has been repeated with much better precision. Figures 26 and 27 show recent results withcut Coulomb corrections. No violation is indicated, contrary to previous indications. The understanding 
of $\pi-D$ scattering is fundamental because it represents a pion interaction with the simpiest nucleus. Also $\pi^{+} d$ scattering yields the D-state probability of the deuteron if the polarization of the recoil deuteron is measured. Some theoretical models of $\pi-d$ scattering predict dibaryon resonances. Figure 28 shows the experimental arrangement for measuring the polarization of the recoil deuteron.

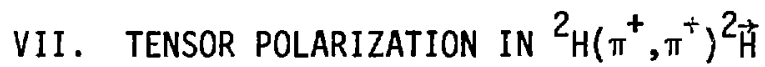

A ${ }^{3}$ He polarimeter is used. ${ }^{3} \mathrm{He}(d, p)^{4}$ He gives proton asymmetry for polarized deuterons. The measurement is sensitive to $T_{20}$ and other polarization moments. It also provides detailed information on the most basic $\pi$-nucleus interaction. Polarizations at large angles are sensitive to specific features of the reaction mechanism. For example, the tensor polarization $T_{29}$ is sensitive to effects of pion absorption on the elastic-scattering amplitude and the D-state probability in the deuteron.

Theoretical work is premised on the impulse approximation, exact three-body calculations, and the $D^{*}$ model, which assumes that the elastic-scattering amplitude is describable in terms of intermediate $\pi-N$ states. From this viewpoint the deuteron provides the simplest means for studying the $\Delta-N$ interaction.

The most complete description will come from three-body calculations. The Fadéev method can solve the $\pi N N$ system exactly. Because the nuclear structure of the deuteron is relatively simple, this approach looks promising.

VIII. DOUBLE-DIFFERENTIAL CROSS-SECTION MEASUREMENT FOR THE REACTION $\vec{p}+d \rightarrow$ $3_{\mathrm{He}}+\mathrm{mm}$ (missing mass)

This is an old problem. Enhancement of multipion production near $2 \pi^{0}$ missing mass in the isospin singlet channel is not consistent with the $\pi-\pi$ scattering length. Figure 29 displays res' 1 ts for the above reaction. Possible explanations are

(1) multistep processes, for e.sumple, $\Delta$-isobar production;

(2) formation of intermediate $\Delta^{*}$; and

(3) modification of phase space because of dependence of the production mechanism on the ${ }^{3} \mathrm{He}$ form factor.

IX. POLARIZED AND UNPOLARIZED PROTON-NUCLEUS SCATTERING

A major goal of proton-nucleus scattering is to test microscopic theories of scattering. For elastic scattering these theories invoke the impulse approximation to describe proton-nucleus scattering in terms of the free proton-nucleon 
interaction. It is then possible to obtain nuclear structure information from the data.

Even though elastic scattering is mainly diffractive, one cüi extract detailed information about neutron density distributions including rms values, $n-p$ rms radius differences, and radial shapes. These can be compared to Hartree-Fock predictions.

The emergence of an energy dependence for the above values would indicate that the impulse approximation is not completely valid.

Much of our p-nucleus data are taken with the High-Resolution Spectrometer (HRS) (Fig. 30). This is an energy-loss spectrometer with a resolution of $50 \mathrm{keV}$ at $800 \mathrm{MeV}$. These data (Fig. 31) are quite remarkable. The cross sections span six or seven orders of magnitude, the precision is quite high, and the angular resolution is very good. The frequency of oscillation is essentially constant and results from the fact that the dominant effect is geometric. The oscillation frequency relates to the nuclear size. The exponential fall-off is due to the diffuseness of the nucleus at the surface. Precise data are essential because information on nuclear structure is derived from the deviation of the experimental data from what one would obtain on purely geometric (diffraction scattering) arguments.

Elastic scattering and analyzing power measurements make possible systematic studies of the adequacy of microscopic descriptions of $p-n$ scattering. The eventual goal is to describe data with no adjustable parameters. The $p+{ }^{208} \mathrm{~Pb}$ data were fit by impulse approximations that use nucleon-nucleon amplitudes obtained from phase-shift analysis of free nucleon-nucleon data.

It was necessary to include the electromagnetic one-photon-exchange contribution to the proton-proton spin-orbit ampiitude, as well as the tar jet-nucleon correlation contribution to the second order optical potential. Wher these corrections are included the position of the maxima and minima in the predicted analyzing power (Fig. 32) shifts into alignment with the data at all forward angles where previous calculations have failed. Figure 32 shows analyzing power data compared to Kerman-McManus-Thaler (KMT) predictions, but including the effect of one-photon-exchange contributiors to the electromagnetic spin-orbit amplitude, with two-body amplitudes from R. Arndt's phase-shift solution.

The spin-orbit term is important in the first-order optical potential. It affects theoretical predictions in the same way as do nucleon-nucleon correlations, so not much can be said about the latter until the spin-dependent 
amplitudes are determined. Fortunately, asymmetry data at forward angles are not sensitive to correlations, only to the spin-orbit potential, Spin-dependent effects are dramatic for large nuclei. Figures 33 and 34 show results for $90,92 \mathrm{Zr}$. A collective model DWBA analysis provides good fits to these data if the spin-orbit potential is deformed. Dashed curves are without spin-orbit deformation. Nuclear-shape symmetries have important impact on nuclear-level ciensities.

A major goal of proton elastic-scattering distributions is the investigation of nuclear-matter distributions as well as details of nucleon-nucleon scattering amplitudes in nuclear matter.

The p-nucleus scattering, together with $\pi^{ \pm}$-nucleus scattering and electron scattering, will yield nucleon-density distributions.

KMT optical-potential analys is has been used to provide a reasonable description of data. We do not yet have good agreement of neutron rms radius with theoretical prediction when $\mathrm{N}-\mathrm{N}$ amplitudes and impulse approximation are used.

The prospect of measuring neutron distributions in nuclei is now in sight. Figures 35 and 36 are examples of the kinds of proton-scattering data that provide the sensitivity required. Recent results indicate an energy dependence of the apparent neutron distributions.

Determination of scattering amplitudes is, of course, an important objective. A measurement was made of the spin-rotation parameter "Q" in the scattering of 0.5-GeV polarized protons from ${ }^{40} \mathrm{Ca}$ (Fig. 37).

$Q=D_{L S} \cos \theta+D_{S S} \sin \theta$.

$D_{L S}$ and $D_{S S}$ are triple-scattering parameters.

$Q, d \sigma / d \Omega$, and $A_{y}$ yield complete information on the scattering amplitude of the reaction, except for a phase factor. These data help determine neutron-density distributions in the nucleus. Figure 38 shows some calculated neutron-density distribution differences for tne calcium isotopes.

A major question is the reactive content of the optical-model potential. How much of the p-nucleus total cross section is accounted for by the inclusive $\left(p, p^{\prime}\right)$ cross section? This bears on the validity of the assumption, in most multiple-scattering theories, that one can use the free nucleon-nucleon scattering amplitudes to describe nucleon-nucleus scattering. If the impulse approximation is valid at $800 \mathrm{MeV}$, the p-nucleus total reaction cross section should be 
composed of the two-nucleon quasi-elastic $[(p, 2 p)$ or $(p, p n)]$ and quasi-elastic delta-production $(p, N \Delta)$. Inelastic scattering measurements show peaks due to quasi-elastic $\mathrm{p}-\mathrm{N}$ and $\mathrm{p}-\Delta$ scattering that appear to dominate the total nonelastic cross section. Proton + nucleus processes appear similar to proton + nucleon processes.

Data for ${ }^{12} \mathrm{C}$ and ${ }^{40} \mathrm{Ca}$ have similar shapes to that from ${ }^{1} \mathrm{H}$ and ${ }^{2} \mathrm{H}$ suggesting that the nucleon-knockout and delta-production processes account for must of the observations. Angle-integrated cross sections for ${ }^{12} \mathrm{C}$ and ${ }^{40} \mathrm{Ca}$ account for more than $90 \%$ of the tota $1800-\mathrm{MeV} \mathrm{p}+{ }^{12} \mathrm{C},{ }^{40} \mathrm{Ca}$ reaction cross sections.

It begins to appear that quasi-elastic processes can explain $800-\mathrm{MeV}$ pnucleus reaction cross sections. The results appear to justify the use of the free $\mathrm{N}-\mathrm{N}$ amplitudes in the KMT (impulse) approximation.

Proton scattering from ${ }^{12,13} \mathrm{C}$ yielded angular distributions to a momentum transfer $q=7.1 \mathrm{fm}^{-1}$. At this $q$, the $\sigma_{e \ell} \simeq 0.02 \mathrm{mb} / \mathrm{sr}$. Figures $39,40,41$, and 42 illustrate the kind of data obtained. Data analysis used DWBA and coupiedchannels formalism. Multipole moments extracted from optical potentials agree with those of the charge densities obtained from electron-scattering experimentr.

Ericson and his colleagues have suggested that one might detect the onset of nuclear-structure effects that precedes the increase of nuclear-matter density to a large enough value to form the pion condensate originally hypothesized by Migdal. It has been suggested that a "nuclear critical opalescence" nay occur which would reveal itself in the excitation of unnatural-parity states in complex nuclei.

These states have been looked for in ${ }^{12} \mathrm{C}$. An attempt was made to detect critical opalescence, the precursor to a pion condensate, by inelastic proton scattering at $800 \mathrm{MeV}$ to the ${ }^{12} \mathrm{C}, 15.11-\mathrm{MeV}$ state. Figures 43 and 44 show the results.

At $13.95^{\circ}$ the momentum transfer is $1.8 \mathrm{fm}^{-1}$, where the maximum cross section has been predicted if critical opalescence occurs. However, the 15.11-MeV state is less strongly excited than the $12.71-\mathrm{MeV} \mathrm{1}^{+}, \mathrm{T}=0$ state, which is not expected to be enhanced. The absence of a secondary maximum shows that no iarge precursor effects due to pion condensates are present for this state with $800-$ MeV protons.

The search for nuclear critical opalescence will be continued in the reaction ${ }^{40} \mathrm{Ca}\left(\pi^{+}, 2 \gamma\right)$ at $\mathrm{T}_{\pi}=55 \mathrm{MeV}$, which will measure $\mathrm{d}^{2} \sigma / \mathrm{dqdx}$. 
Momentum transfer $q$ will be in the range 0.5 to $2.0 \mathrm{~m}_{\pi^{*}}$. The range of the photon eriergy-sharing parameter $X$ will be from -0.75 to 0.75 .

The above reaction is hoped to be sensitive to medium modifications to the pion propagators present in the matrix elements. The effect should be most pronounced for $x=0$ and $q \cong 2.2 m_{\pi}$ (Ericson and Delorum). Theoretical efforts are under way to calculate the cross section using the distorted-wave impulse approximation for one-nucleon processes and including medium modifications to the propagators.

\section{$X$. SEARCH FOR DELTAS IN A COMPLEX NUCLEUS BY A RADIOCHEMICAL TECHNIQUE} (Turkevich, Fig. 45.)

Almost all inelastic proton cross sections can be described in terms of quasi-elastic $(p, p N)$ and $p, p \Delta)$ processes in complex nuclei. Deltas are defined as excited states of nucleons with mass $1232 \mathrm{MeV}$ and also higher mass states, which can exist in charge states $+2,+1,0,-1$. These systems are endoergic and therefore can only exist as virtual states in normal nuclear matter. Processes such as $\left(p, p \pi^{+}\right)$in compiex nuclei can be described as either due to the elastic collision of a proton with a pre-existing virtual $\Delta^{++}$or the collision of a proton with a virtual pion cloud. It is possible that $\Delta$-knockoui can be unambiguously observed by looking for the results of the collision of an incoming neutron with a $\Delta^{++}$inside nuclear matter resulting in the liberation of $\Delta^{++}$and leaving behind a very low-energy neutron. An experiment was performed to look for

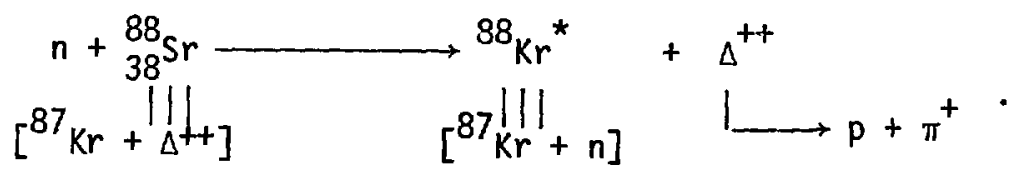

A one-step pion.prodiction mechanism cannot convert ${ }^{88} \mathrm{Sr}$ to ${ }^{88} \mathrm{Kr}$. Two-step processes are expected to be at the less-than-microbarn cross-section level. Steady-state presence of $1 \% \dot{\Delta}^{++}$in ${ }^{* *}$ Sr would provide an 8 - $\mu$ b cross section for the production of ${ }^{88} \mathrm{Kr}$.

$X I$. MUONIC $X$-RAY SPECTRA: OSMIUM ISOTOPES IN THE 2p-1s TRANSITION REGION

Muonic $x$-ray experiments yield information about nuclear charge and matter distribution. Figure 46 shows the Stopped Muon Channel (SMC). Muonic atoms provide the possibility of high precision x-ray measurements of hydrogen-like systems to check the extent to which the muon obeys QED corrections, to look for the existence of new types of interactions, and to test $\mu$-e universality. 
One can test the vacuum polarization in QED to better than one part in a thousand. Tests of QED vacuum polarization corrections come from $\left(\mu^{-}{ }^{3} \mathrm{He}\right)^{+}$and $\left(\mu^{-}{ }^{4} \mathrm{He}\right)^{+}$. Tests of the $\mu-e$ interaction come from studies of the neutral muonic hel ium a iom and of muonium.

ine spectra of transitional nuclai, Fig. 47, show (1) line pattern shifts to lower energy with increasing mass due to increase in nuclear radius and (2) hfs pattern changes in systematic ways. An additional 1 ine in ${ }^{188} 0 \mathrm{~s}$ is due to muonium-nuclear resonance. Precision measurements were made of the deformed osmium isotopes. Equivalent Barnett radii and isotope shifts were determined; also isomer shifts for the first excited $2^{+}$states. Generalized E- 2 moments of charge distribution were obtained and conventional electromagnetic moments were determined.

Osmium nuclei 1 ie in the shape-transitional region between strongly deformed rare-earth nuclei and the spherical ones near ${ }^{208} \mathrm{~Pb}$. Excited-state quadrupole moments are determined from the hyperfine spectra of muonic atoms.

Muonic x-ray spectra, together with electron scattering, yield charge and matter form factors:

(1) ground-state monopole charge parameters,

(2) isomer shifts of excited nuclear states, and

(3) multipole charge parameters.

Figure 48 shows charge distribution for ${ }^{165_{\text {Ho }}}$ for a cut through the poles. Solid and dashed lines use different models. One obtains E-2 and E-4 moments as well as radial moments. The nuclear charge radi $i$ deduced from muonic $x$-ray measurements show the following systematics, Fig. 49.

(1) Large increase in charge radius at the beginning of a new shell.

(2) Almost linear decrease in radii at shell closure. Sometimes radii differences become negative.

(3) Almost no dependence on nuclear charge for the radi i differences.

(4) Constant decrease of radius, as a function of neutron number, for ${ }^{78} \mathrm{Kr}$ to ${ }^{86} \mathrm{Kr}$.

Muonic $x$-ray transitions together with electron scattering give, in a model independent way, not only charge radii but also higher radial moments $\left\langle r^{2}\right.$, and $\left\langle r^{4}\right\rangle$. These then permit determination of the electron charge density at the nucleus. This, in turn, permits determination of absolute radii of unstable nuclei from optical isotope-shift data. 
XII. PIONIC ATOMS

Figure 50 shows an interesting effect--the so-called nuclear resonance effect. It has been used to determine the nature of the $\pi$-nucleus $p$-wave interaction.

XIII. $\pi^{0}$ SPECTROMETER

Pion Single Charge Exchange

A $\pi^{0}$ spectrometer, Figs. 51 and 52, with a resolution of better than $2 \mathrm{MeV}$ at $200 \mathrm{MeV}$ has been developed. It measures the direction and energy of the $\pi^{0}$ produced in a single charge exchange reaction. Reactions studied are $\pi^{+}+(A, Z) \rightarrow$ $\pi^{0}+(A, Z+1)$ and $\pi^{-}+(A, Z) \rightarrow \pi^{0}+(A, Z-1)$. Most experiments have studied $\left(\pi^{+}, \pi^{0}\right)$ to isobaric analog states.

Figure 53 shows the fundamental $\pi^{-} p-\pi^{0}$ reaction. Figure 54 shows some isobaric analog transitions. Cross sections appear to be describable by a socalied peripheral model in which the reaction is dominated by the neutron-proton density difference (isovector density) at the point in the nucleus where the isoscalar density (neutron plus proton density) has dropped off to $10 \%$ of its central density.

XIV. FARE DECAY OF PIONS

The $\pi^{0}$ spectrometer has also been used to study the beta decay of the pion. This is part of a program to push conservation laws as far as possible. CVC hypothes is is basic to weak-interaction theory. Nuciear beta decay data supports CVC hypothesis, but ambiguities occur due to nuclear-structure effects. A very sensitive test of CVC can be made by precise measurement of the branching ratio for $\pi^{ \pm} \rightarrow \pi^{0}+e^{ \pm}+\nu$, Fig. 55, which theory predicts to be $1.048 \pm 0.008 \times 10^{-3}$. Preliminary results are in agreement with CVC. The uncertainty is $\pm 3 \%$.

We have searched for the reaction $\pi^{0} \rightarrow 3 \gamma$ to test charge conjugation. $\pi^{0}$ has charge conjugation quantum number +1 as shown by its decay into two photons, each with $C=-1$. Thus $\pi^{0} \rightarrow 3 \gamma$ is forbidden by $C$ invariance. We do not see it.

XV. PION-NUCLEUS INTERACTIONS

One of our majoi programs is concerned with using the pion as a nuclear probe. A major goal of this work is to understand m-nucleus scattering and $\pi$ absorption in the nuclear medium. An important aspect is the behavior (formation, propagation, and decay) of the $\Delta(3,3)$ isobars in nuclei. $\Delta(3,3)$ is the 1232-MeV resonance whose width one wants to determine. This quasi-elastic portion of the 
inelastic scattering is of particular interest. Single-arm experiments and coincidence experiments can be used to study the single-nucleon knockout process. The mechanism of pion absorption in nuclei can be explored by way of correlations of emitted particles. One test of pion absorption is provided by $\pi^{+} d \rightarrow 2 p$ and $i$ ts inverse. This reaction yields data on higher partial waves and dibaryon resonances. The pion can illuminate properties of the nucleus in complementary fashion to other probes because of the following properties.

(1) The pion is an isospin triplet. This property enables single and double charge exchange. It also means that $\pi^{-} n$ and $\pi^{+} p$ amplitudes are enhanced over the $\pi^{-} p$ and $\pi^{+} n$ amplitudes at energies near the 3,3 resonance.

(2) Because its mass is one-seventh that of the nucleon, recoil corrections in the analyses of pion-nucleon collisions are small.

(3) The pion has zero spin and is therefore a boson that can be created or destroyed singly. The zero spin simplifies theoretical analysis of $\pi$-nucleus interactions.

(4) $\pi$-nucleus scattering amplitudes exhibit sharp resonances, leading to strong scattering and/or absorption at certain energies. The 3,3 resonance is narrower than for $\pi-N$ scattering.

(5) $\pi^{+}-$and $\pi^{-}$-induced reactions car be performed in an experimentally symmetric fashion. Pure proton and neutron transitions are observed in ${ }^{14} \mathrm{C}$, as is isospin mixing.

(6) It is possible to produce pionic atoms with "ticklish" nuclei.

Recent data yield the surprising result that low-energy pion interactions in nuclei are enormously modified by the nuclear mediun, leading to much larger mean-free paths than we would deduce from free pion-nucleon cross sections. Also, giant resonances are weakly excited by $80-\mathrm{MeV}$ protons, again attesting to the weakness of the effective $\pi-N$ interaction in nuclei for low-energy pions.

Pion-induced two-nucleon emissions from compiex nuclei show large ratios of the yields of two-neutron-out products compared to those resulting from the emission of a neutron and a proton. This may indicate that the nuclear skin is being probed and that it is more neutron-rich than the interior.

For energies near the $(3,3)$ resonance, the surface is probed; $\pi^{+}$and $\pi^{-}$ differentiate between neutrons and protons, as we shall see.

Figures 56, 57, and 58 illustrate the kind of data attainable by inelastic $\pi^{ \pm}$scattering. Pure neutron and pure proton transitions are measured. Such 
states, observed in ${ }^{14} \mathrm{C}$, peak at large $\mathrm{q}(\sim 275 \mathrm{MeV} / \mathrm{c})$, so they are probably high-momentum states. Strong correlation of proton and neutron transition amplitudes are observed. At forward angles the difference of $\pi^{ \pm}$elastic scattering cross-sections can be used to determine the real part of the nuclear scattering amplitude (for pion scattering on isoscalar nuclei). Using the optical theorem, total cross-section measurements yield the imaginary part of the nuclear forward scattering amplitude.

The $\left(\pi^{+}, p\right)$ and $(p, d)$ cross sections and angular distributions look quite similar, indicating similarity in the underlying mechanism.

Some interesting results have been observed in the scattering of $\pi^{+}$and $\pi^{-}$ by ${ }^{3} \mathrm{He}$ and ${ }^{4} \mathrm{He}$.

(1) The differential cross section for ${ }^{4} \mathrm{He}\left(\pi^{-}, \pi^{+}\right)$measured at Berkeley is too low by a factor of 100 . This erroneous result has for 10 years frustrated attempts to explain double charge exchange.

(2) The ratio of $\left(\pi^{-}, p\right)$ to $\left(\pi^{+}, p\right)$ at $200 \mathrm{MeV}$ is $1: 20$, and $1: 30$ for ${ }^{3} \mathrm{He}$ and ${ }^{4} \mathrm{He}$. The isobar model for $\pi N N \rightarrow N p$, whereby $\pi+N_{1} \rightarrow \Delta$ and $\Delta+N_{2} \rightarrow N p$, predicts $1: 10$ and $1: 20$. The $\left(\pi^{-}, p\right)$ cross section is smaller for ${ }^{3} \mathrm{He}$ than for ${ }^{4} \mathrm{He}$.

Pion charge exchange on complex nuclei has yielded unexpected results. Single charge exchange $(S C X)$ can, of course, be achieved by beta decay and hadronic reactions. But double charge exchange ( $D C X)$ is most conveniently achieved with pions, even though the cross sections are low (microbarns).

Because pions have isospin $T=1$ and three charge siates, the reactions $\left(\pi^{ \pm}, \pi^{\mp}\right)$ are marvelously clean $D C X$ reactions, leading to nuclei far $f \cdots \cdots$, the 1 ine of stability. Al though DCX probably consists of two successive steps of $S C X$, the physics content is unique. This comes about because we can access isospin states by exciting $T=T_{Z}+2(\Delta T=2)$ double analog states. In addition, DCX is an excellent probe for studying nucleon-nucleon cor:alations inside nuclei. It is also sensitive to nuclear-matter distributions because of its selectivity to the type of nucleons involved. Because the reaction involves double isospin flip of the projectile, the isospin structure of the nucleus may be involved. $D C X$ also yields information on the isovector aspects of nuclear structure since, in the simplest picture, $\left(\pi^{+}, \pi^{-}\right)$reactions take place on the $(N-Z)$ extra-core neutrons. 
XVI. PION DOUBLE CHARGE EXCHANGE

Figure 59 shows the Low Energy Pion Channel (LEP) that has been used for pion double-charge-exchange measurements.

Pion double charge exchange permits mass measurements (Figs. 60 to 63) of proton- and neutron-rich nuclei and provides a sensitive test of models of the pion-nucleus interaction. Targets were ${ }^{9} \mathrm{Be},{ }^{12} \mathrm{C},{ }^{13} \mathrm{C},{ }^{16} 0,{ }^{24} \mathrm{Mg}$, and ${ }^{36} \mathrm{~S}$ for $\left(\pi^{+}, \pi^{-}\right)$reactions.

Double-charge-exchange results obtained are not explainable on the basis of single-particle wave functions for initial and final states. Nuclear structure enters the picture, which is both good news and bad news.

One dramatic result is that the ground-state transition cross sections to nondouble-isobaric-analogue states are just as large as to the double-isobaricanalogue states (DIAS). The latter were predicted to dominate because of the simplicity of the reacrion mechanism--a double raising and lowering of the isospin operator.

The Holy Grail remains the determination of the complete nuclear wave function. The fact that there are, in addition to nucleonic components, also pionic and isobaric components does not make the job easier.

Systematics are starting to emerge in double charge exchange yielding doubleanalogue and non-analogue transitions. Nucieon correlations inside nuclei are being probed. Systematic trends are seen as a function of target isospin and mass and pion kinetic energy. For ${ }^{16,18} 0$ and ${ }^{24,26} \mathrm{Mg}$, the transitions to the DIAS of the $T=1$ nuclei have the same shape. Non-analogue ground-state transitions from $T=0$ nuclei also exhibit similar structures. Strong cancellations of neutron and proton transition amplitudes are observed in inelastic scattering from ${ }^{14} \mathrm{C}$.

Near the $(3,3) \pi$-resonance, the ratio $R=\sigma \pi^{+} / \sigma \pi^{-}$froin free protons (neutrons) is $\sim 9(1 / 9)$. Therefore, comparison of $\pi^{+}$and $\pi^{-}$inelastic scattering from nuclei provides a method of determining the proton and neutron components of inelastic transitions. Cross-section ratios near the free- $\pi$ values are expected for pure proton or neutron particle-hole $(p-h)$ excitations if the impulse approximation is valid. Deviations from $R=1$ for $N=Z$ nuclei arise from charge symmetry violations, that is isospin mixing due to coulomb force, and have been observed. In the case of ${ }^{12} \mathrm{C}$ and ${ }^{16} 0$, they have been interpreted as arising from transitions to an isospin-mixed doublet in ${ }^{12} \mathrm{C}$ (and a triplet in ${ }^{16} 0$ ). The free $\pi$-neutron value of $R \sim 1 / 9$ was detected only for a transition 
in ${ }^{13} \mathrm{C}$ and interpreted as the result of a pure neutron particle-hole trarsition to a stretched state. R significantly greater than 9 has been observed for ${ }^{14} \mathrm{C}$ and reflects dramatically the shell-model structure of the transitions. It is due to strong cancellation of the proton and neutron transition amplitudes.

In summary, we are addressing nuclear structure along the lines shown in Fig. 64. One aim is to obtain matter distributions.

XVII. WEAK INTERACTIONS AND UNIFICATION THEORIES

Figure 65 shows our goals for this major program. The normal decay of the muon permits study of the weak interaction involving purely leptonic processes. Although measurements to date favor a $(V-A)$-type interaction, they do not exclude considerable scalar, pseudoscalar, and tensor contributions. A high-precision experiment is being mounted to measure the electron spectrum in the allowed beta decay of the muon (Anderson). The experiment will provide very precise Michel parameters for this purely leptonic beta decay. A time-projection chamber will be used. This experiment will shed light on the V-A character of the weak interaction.

An experiment already performed involved observation of the neutrinos from muon decay. It was designed to determine to what extent the lepton-number conservation laws might be multiplicative rather than additive. Figure 66 shows a schematic of the experiment.

This experiment searched for $\bar{\nu}_{e}$ from $\mu^{+}$decay. A six-ton water Cerenkov detector filled with $\mathrm{H}_{2} \mathrm{O}$ was used to look for the $\bar{\nu}_{e} \mathrm{p} \rightarrow \mathrm{e}^{+} \mathrm{n}$ reaction and in $\mathrm{D}_{2} \mathrm{O}$ to measure the reaction $\nu_{e} D \rightarrow p e^{-} p$. Three important results were obtained.

$$
\begin{aligned}
& \text { 1. } \mu^{+} \rightarrow \mathrm{e}^{+}+\nu_{\mathrm{e}}+\bar{\nu}_{\mu}-\text { Additive Law } \\
& \mu^{+} \rightarrow \mathrm{e}^{+}+\bar{\nu}_{\mathrm{e}}+\nu_{\mu}-\text { Multiplicative Law } \\
& \\
& \frac{\left[\mu^{+}+\bar{\nu}_{\mathrm{e}}\right]}{\left[\mu^{+} \rightarrow \nu_{\mathrm{e}}\right]}<0.09 .
\end{aligned}
$$

2. Inverse solar pe $p$ reaction cross section $\left(\nu_{e} D \rightarrow p e^{-} p\right)=5 \times 10^{-41} \mathrm{~cm}^{2}$ in good agreement with theory.

3. No neutrino oscillation from $\bar{\nu}_{\mu} \rightarrow \bar{v}_{e}$.

Experiment 225 will measure $v_{e}$-e elastic scattering, will provide a purely leptonic determination of $\theta_{\omega}$, and is also sensitive to the interference, both in 
sign and magnitude, between charged- and neutral-current diagrams. This exirariment uses a 13-ton detector comprised of 9 tons of plastic scintillator and 4 tons of polypropylene flash chambers and will measure scattered electrons. The presence of hydrogen will also permit the search for $\bar{v}_{e}$ from the oscillation channel $\bar{v}_{\mu} \rightarrow \bar{v}_{e}$, the reaction being $\bar{v}_{\mathrm{e}} \mathrm{p} \rightarrow \mathrm{e}^{+} \mathrm{n}$. For $\Delta \mathrm{m}^{2}>0.3 \mathrm{ev}^{2}$, the rate would be 100 events/day. Cosmic-ray background will be 4-45 per day.

XVIII. UNIFICATION OF WEAK AND ELECTROMAGNETIC INTERACTIONS

A large effort is being devoted to studies relating to gauge theories. Gauge models of weak interactions do not, in general, conserve fermion flavors. We must therefore look for such nonconservation.

Exact conservation laws, such as for energy and momentum, are related to space-time translations and rotations. Conservation of electric charge is related to the gauge invariance of a field and its associated massless gauge boson.

Unification of weak and electromagnetic interactions by non-Abelian gauge theories led to discoveries of neutral currents. However, the exact structure of the gauge model is not established. Specifically, in some gauge theories, muon and electron number conservation are not constrained to be exact. Conservation laws for electron number, muon number, and baryon number may only be approximate within the framework of unified theories. Exact global symmetries may be an untenable indulgence.

Muon-number violation limits may provide new constraints on gauge models.

There is a growing belief that there can be no massless gauge bosons associated with lepton and baryon numbers and that these quantities are not exactly conserved. Violation of baryon number conservation could be detected by proton decay or neutron-antineutron oscillations. Violation of lepton-number conservation could be detected by rare decay modes of the muon and neutrino oscillations. Gauge models of the electromagnetic and weak interaction and grand unified theories provide reasonable ways to accommodate very sma11, but nonzero. Teptonnumber and baryon-number violations.

Even if lepton number is not conserved, gauge models predict very low rates for lepton-number violating processes, thus requiring major efforts for their detection.

Here is one of the main bridges between nuclear and subnuclear physics-tests of unified gauge theories. Conserved vector current, time-reversal 
invariance (TRI), neutrino oscillations--all of these are involved. Breakdown of TRI implies an electric dipole moment of the neutron. Gauge theories predict it to be very small $\left(\sim 10^{-25} \mathrm{e} \mathrm{cm}\right)$. Norman Ramsey is exploring whether one might be able to make this measurement at LAMPF.

A number of large experiments and facilities are $i: s$ the planning or startup stage.

\section{A. Crystal Box Experiment}

A crystal box will be used to study the rare decay modes of muons, Fig. 67. Inside is a target where $\mu^{+}$stops. The target is surrounded by an eight-layer drift chamber, 36 hodoscope scintillation counters, and 396 sodium iodide crystals. The energy, position, and time of arrival of photons are measured in the $\mathrm{NaI}$. The trajectories of electrons are measured in the drift chamber, and their times of arrival are measured in the hodoscope counters.

NaI will cover four sides of the box. Inside will be a cylindrical drift chamber for track detection that provides accurate electron direction while the NaI gives good energy determination.

Energy resolution 4\% FWHM,

Position resolution $\sim 2 \mathrm{~cm}$ FWHM

Angle resolution $\sim 20 \mathrm{mrad}$ FWHM

Timing resolution $~ 0.7$ ns FWHM

The following rare decays will be searched for.

$$
\begin{aligned}
\mu^{+} & \rightarrow e^{+} \gamma \\
\mu^{+} & \rightarrow e^{+} e^{+} e^{-} \\
\mu^{+} & \rightarrow e^{+} \gamma \quad \text { (Flavor Violating Decay) }
\end{aligned}
$$

Sensitivity will be $10^{-11}$. We will eventually search for $\mu^{+} \rightarrow e^{+} \gamma$, with a sensitivity of $10^{-12}$ (present limit is $10^{-10}$ ).

The crystal box will permit searches for $\mu^{+} \rightarrow \mathrm{e}^{+} \gamma, \mu^{+} \rightarrow \mathrm{e}^{+} \gamma$, and $\mu^{+} \rightarrow \mathrm{e}^{+} \mathrm{e}^{+} \mathrm{e}^{-}$with 1 to 3 orders of magnitude greater sensitivity than heretofore.

\section{B. Neutrino Experiments}

1. Lepton-number conservation

2. $v_{\mathrm{e}} \mathrm{e}$ elastic scattering

3. Neutrino oscillations 
Neutrine mixing yia nonexponential $\pi \rightarrow \mu v$ decay

Neutrino flux $\sim 4 \times 10^{7} \mathrm{vs} / \mathrm{cm}^{2} \mathrm{~s}$. The beam stop provides a neutrino source $50 \mathrm{~cm}$ in diameter. The spectrum comprises $30-\mathrm{MeV} \nu_{\mu}$ from $\pi^{+}$decay and 0 - to $53-$ $\mathrm{MeV} \mu_{\mathrm{e}}$ and $\bar{\nu}_{\mu}$ from $\mu^{+}$decay. This is a clean source for $\nu_{e}$ scattering since $\nu_{\mu}$ and $\bar{\nu}_{\mu}$ are below threshold for meson production. In-flight pion decay would provide 150- to $200-\mathrm{MeV} \nu_{\mu}$ of $5 \times 10^{5} \mathrm{v} / \mathrm{cm}^{2} \mathrm{~s}$ at $50 \mathrm{~m}$ from the production target.

C. A Genera] Neutrino Facility

A general neutrino facility (Figs, 68 to 71 ) is being proposed in conjunction with the proton storage ring (PSR). Neutrino coupling to nuclear matter will be studied. A large cross section for neutrino-nucleus interactions would be of great significance in terms of the cooling of stars. 


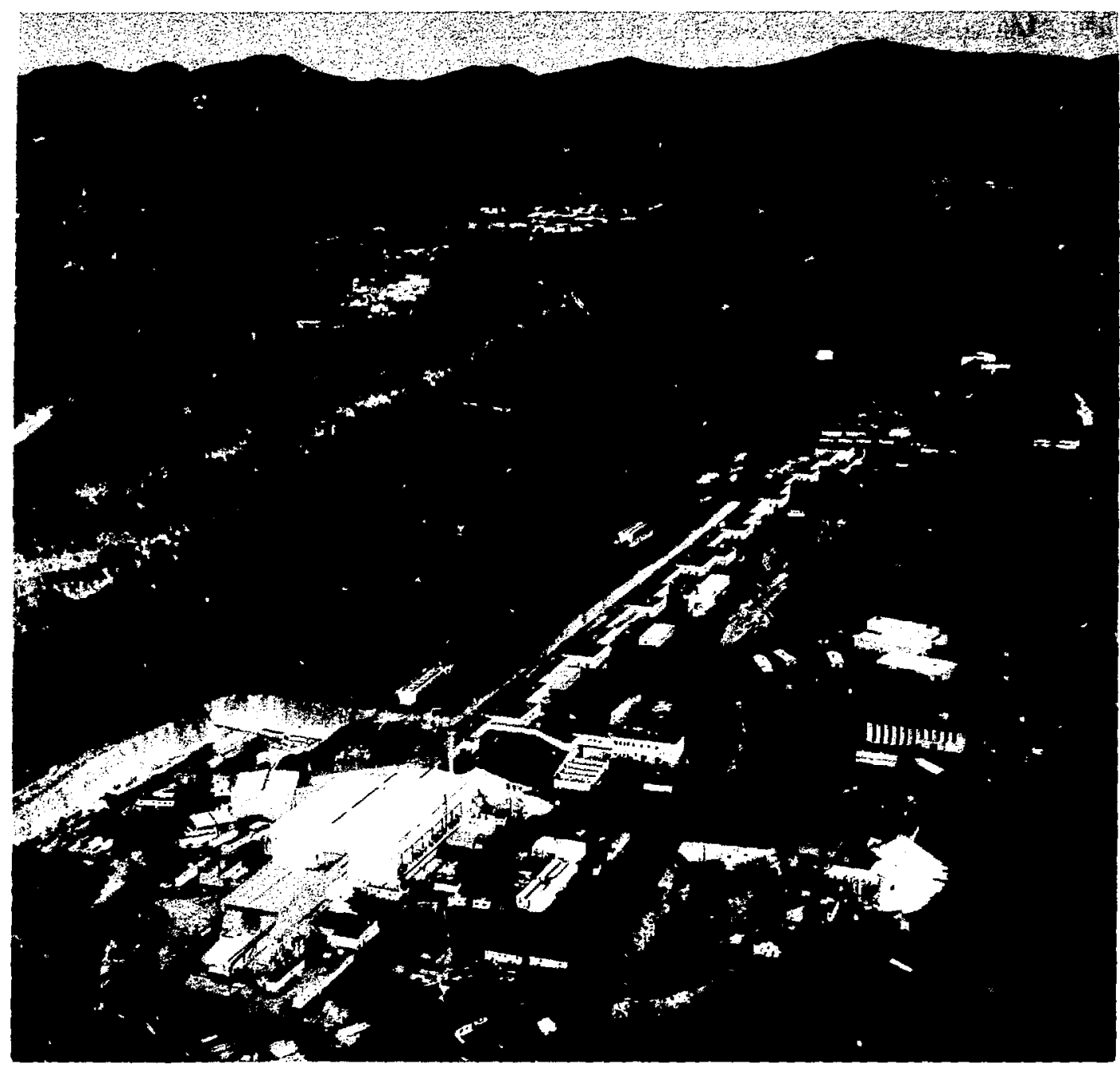

Fig. 1. Aerial view of LAMPF.

\section{REAL-LIFE SITUATION}

We are Using High-Quality Dosms of Polerized and Unpolarized 500-800-1MeV Protons as

1. A mieroscops to see the structure of the Mucleus,

2. A llows to Determine the Force Between Nucleons,

3. A Howitucs to Disintograte Nuclei, Yielding Fragmente lor stuty, and

4. A Mleans to Create Pions and Muons.

Fig. 2. Capabilities of LAMPF. 


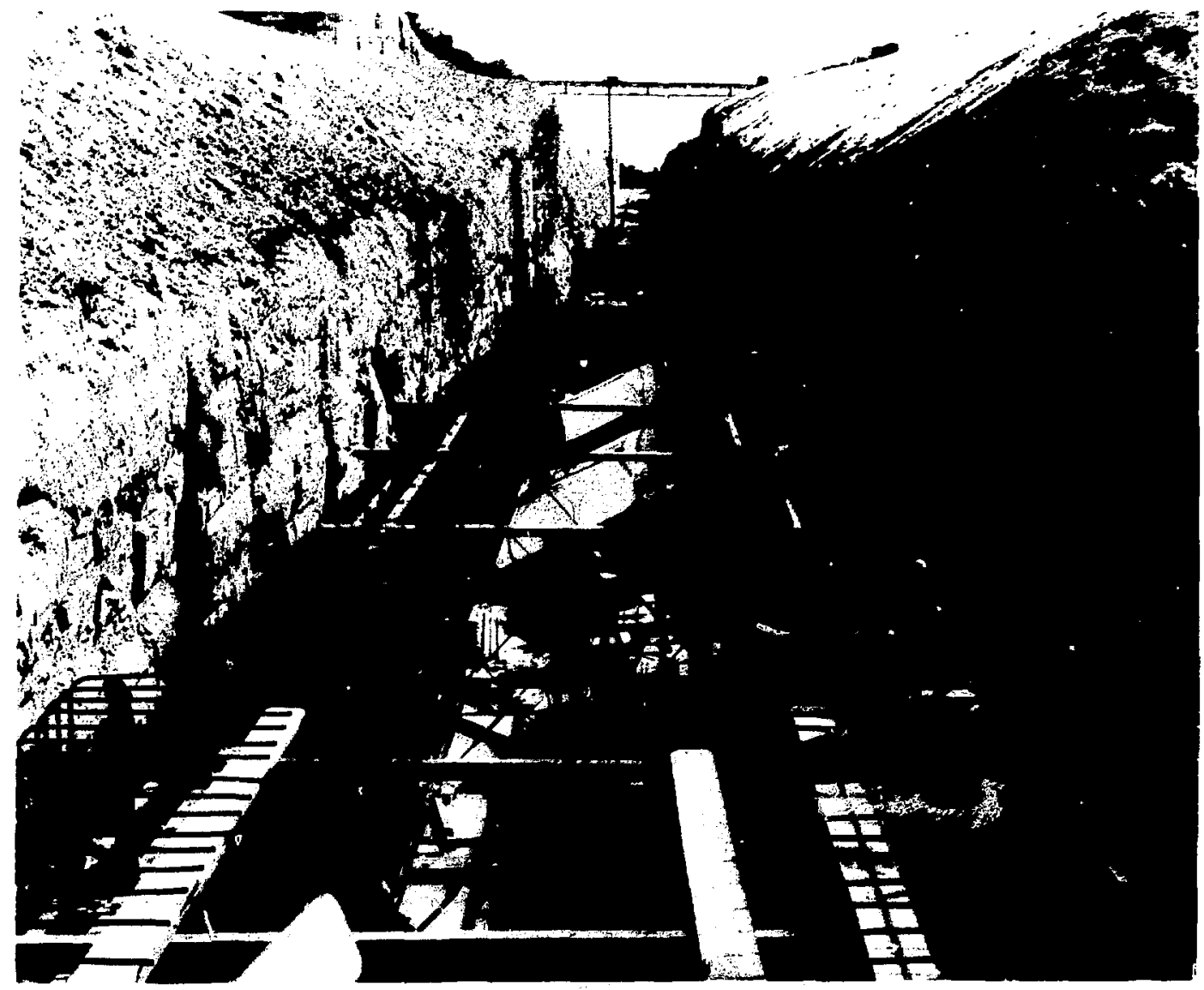

Fig. 3. Excavation of tunnel for the LAMPF accelerator, circa 1969.

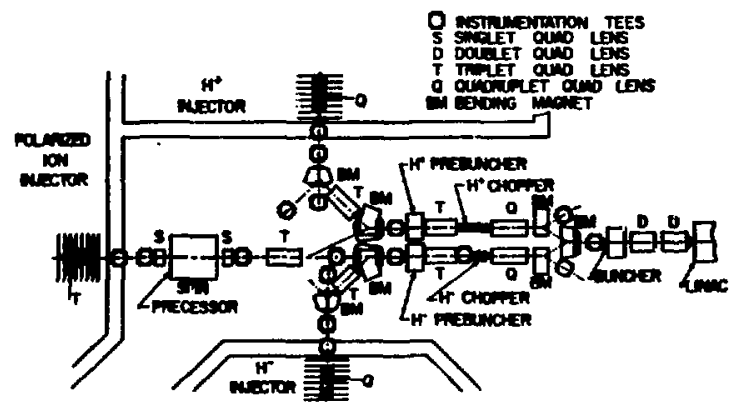

Fig. 4. Schematic of LAMPF injector system. 


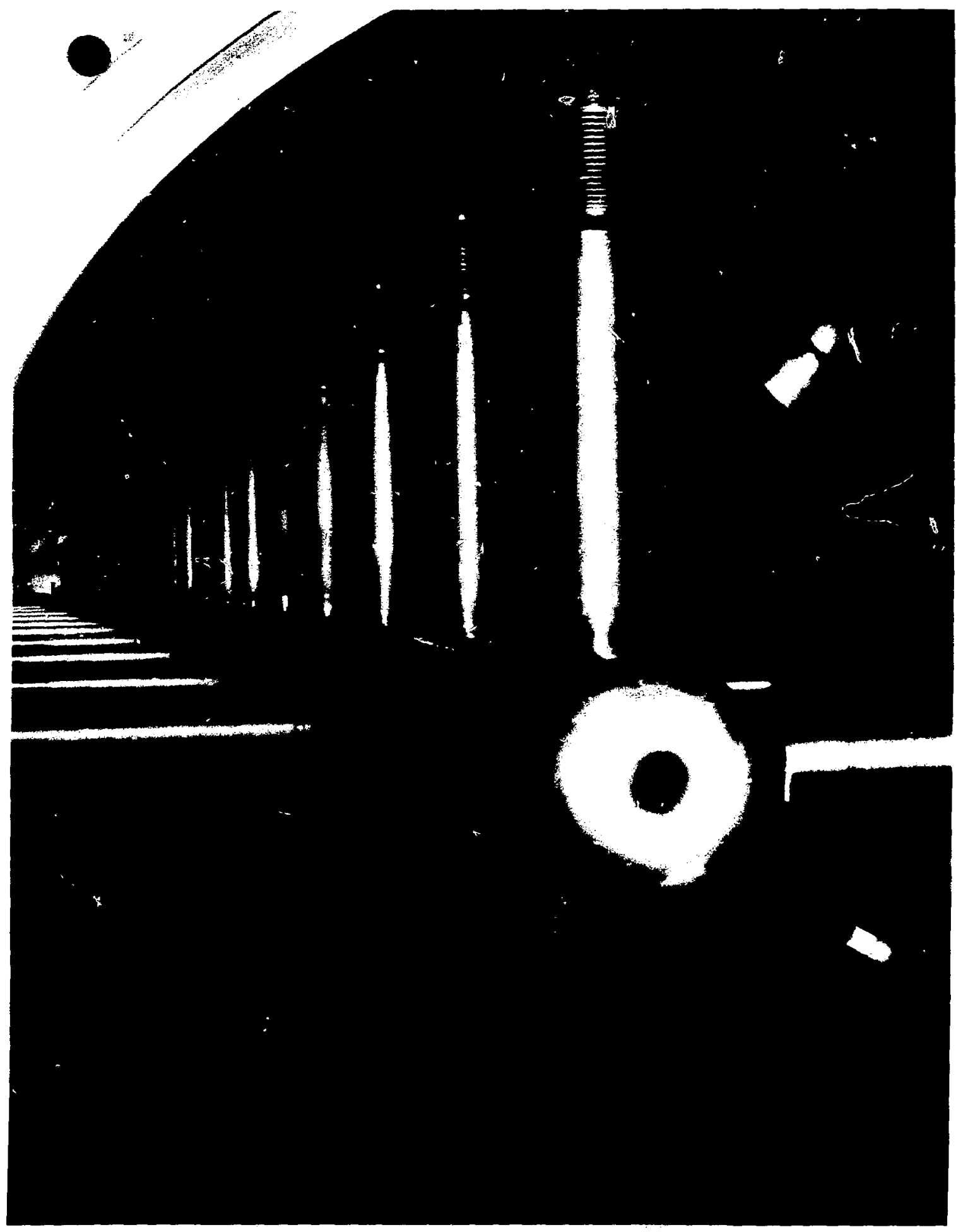

Fig. 5. Drift-tube (Alvarez) Tinac (100 MeV). 


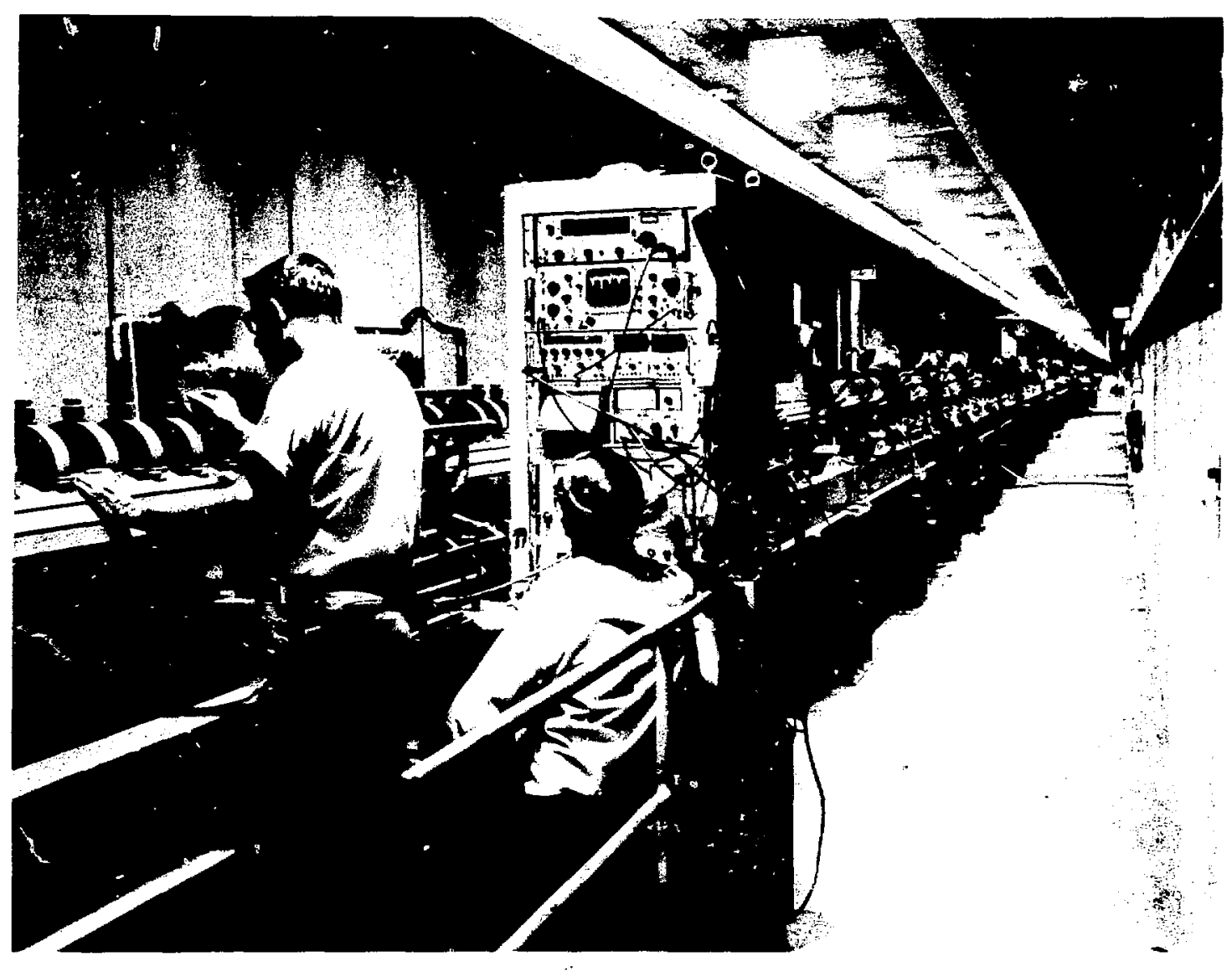

Fig. 6. Side-coupled cavity linac (800 MeV).

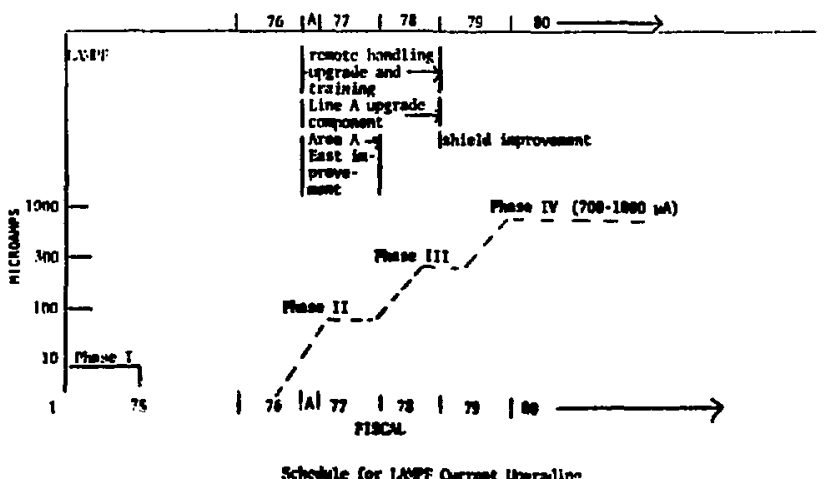

Fig. 7. Beam current as a function of time. 


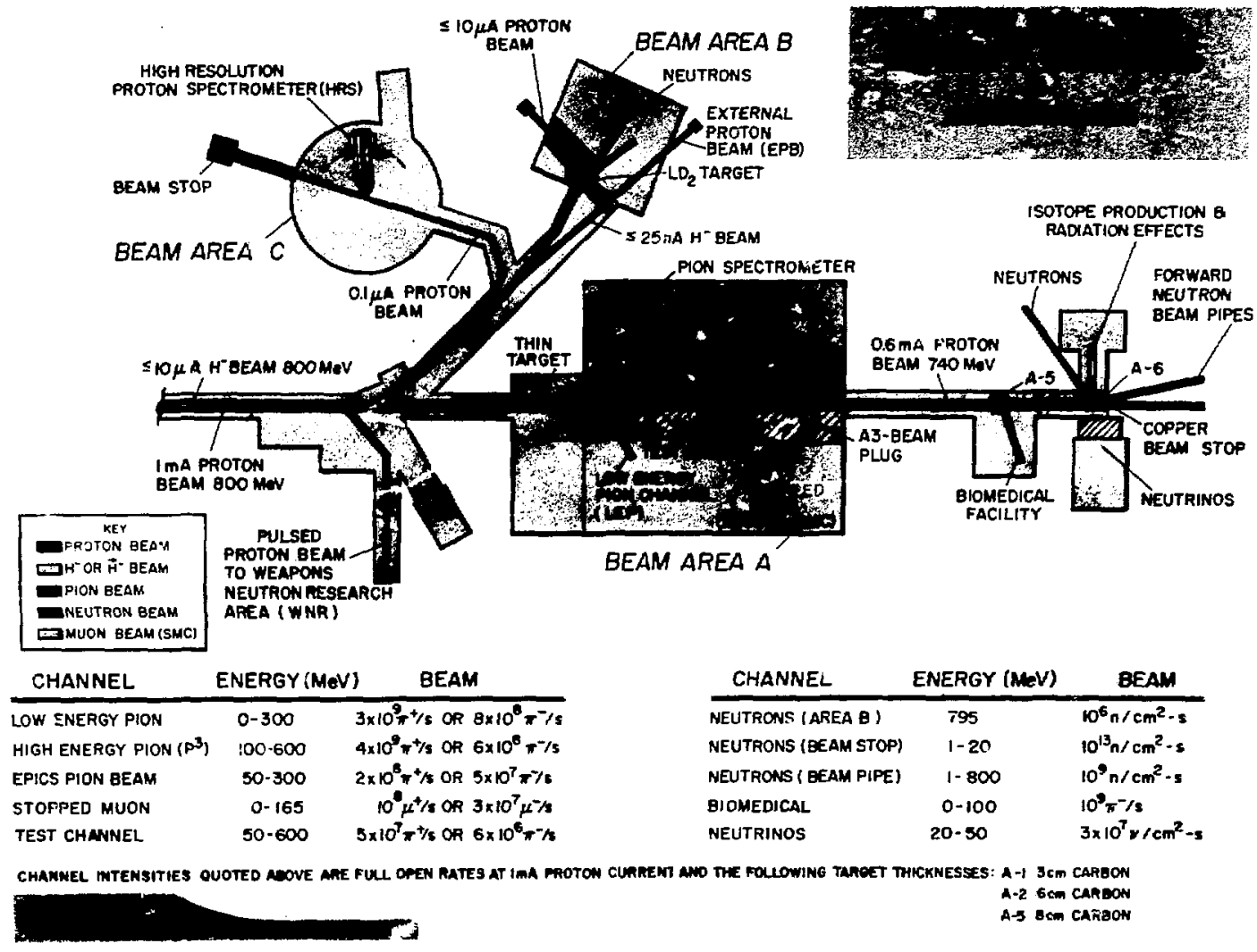

Fig. 8. LAMPF beams and experimental areas. 


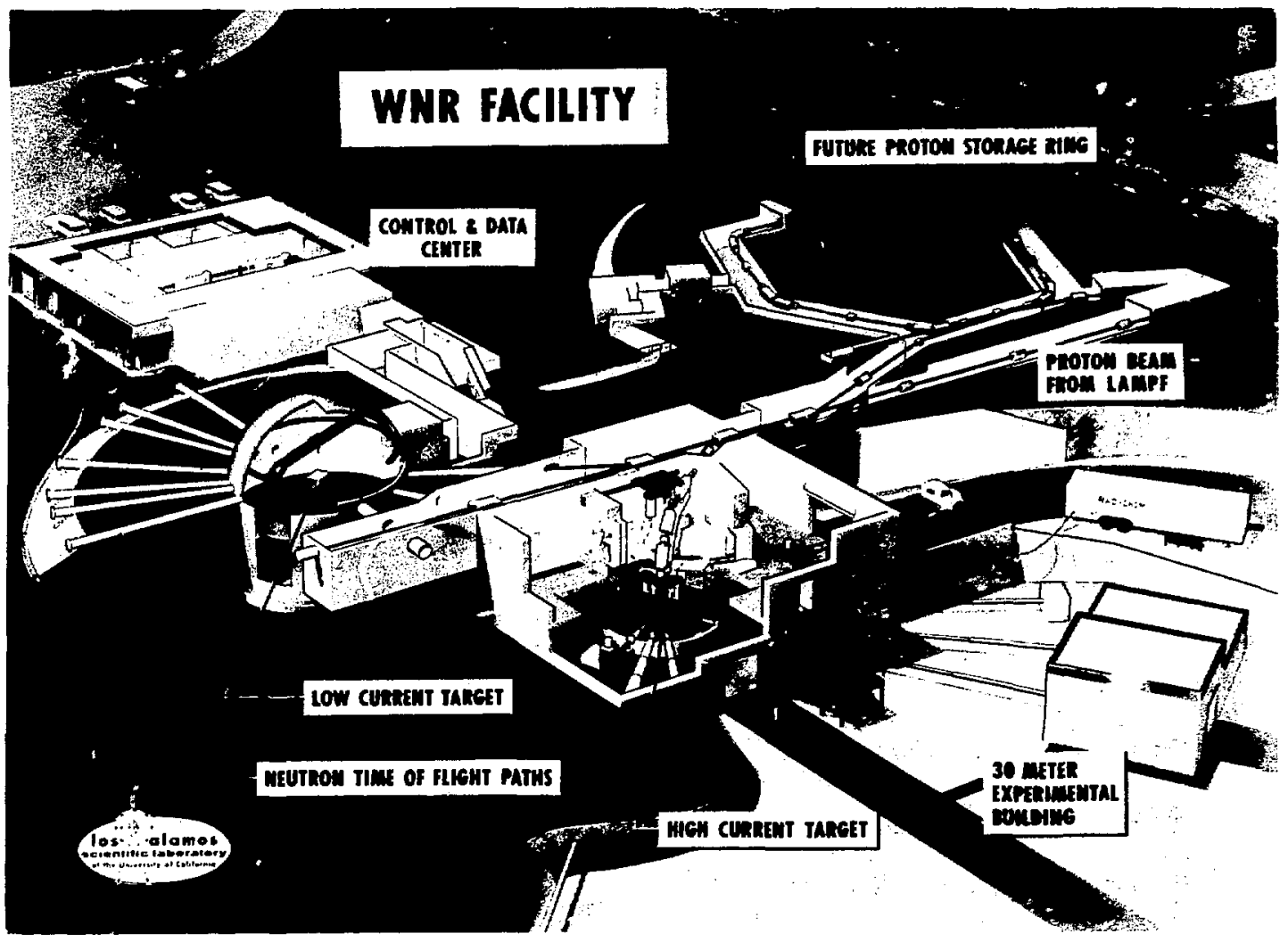

Fig. 9. Weapons Neutron Research (WNR) Facility.

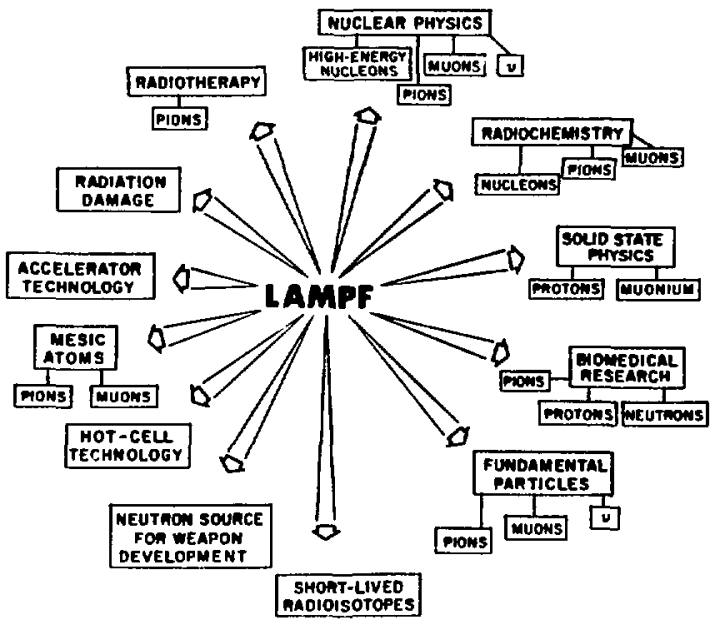

Fig. 10. Goals and objectives of LAMPF.

\section{BREAD \& BUTTER ACTIVITIES}

\section{Forces Between Nucleons}

-Long-flange
- Short-frange
-Origin
- llobe of a nesenance

i1. Teats of QED; Muon Electrodynamics

III. Acestablich Bridge Betmeen Muclem and subnucleser Physics

Fig. 11. Programs presently under way-fundamental experiments. 
IV. Structure and Properties of Muclai

Propertive of Unatebs Nuchi

simple Modes of Motion

-Fen-Perticts stelea

- Clint Reconences

-Perticle Cerrelations

Intermediate and Complex Modes of Motion

- Direct $\rightarrow$ Compound Mucleus Reactions

- Ceneral Applieability to Many-Body

Fig. 12. Programs presently under way-nuclear structure and properties.

IV. (Continued)

Charge and Matter Distributions

Role of Pion in Nuclei

Relationship Between Stafiatical, Collective, and Single-Particle Modes of Motion

Description of Nuclear structure and Reactions in Terms of Two-Nucleon Forces

Fig. 13. Programs presently under way-nuclear structure and properties.

v. Unitication of Woak and Electromagnetic $\mathrm{n}$ Ieractions

\section{TESTS OF:}

- Unified aswe Theories

-Conerved-Vecter-Current Hypetheis

- Timo-Reversel invaricnee

- Nowtrino Coupling to Nublect Matter

Fig. 14. Programs presently under way-grand unified theories.
TESTS OF:

\section{Quantum Mechanics Photodissociation of $\mathrm{H}^{-}$}

Quantum Electrodynamics

$$
\frac{\Gamma\left(x^{0} \rightarrow e^{+} e^{-} \gamma\right)}{\Gamma\left(x^{0} \rightarrow r\right)}
$$

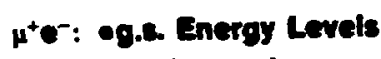
onfe interval

- Muon Magnetic Moment

Conserved-Vector-Curremt Hypothesis:

$$
x^{ \pm} \rightarrow x^{0}+e^{ \pm}+\gamma
$$

C-Invariance:

$$
x^{0} \rightarrow 3 \gamma
$$

Fig. 15. Tests of quantum electrodynamics: rare and forbidden decays of the pion; CVC; properties of muonium.

SHAFT TO

STEPPING MOTOR UPSTEEAM ASSEMBLY MAGHET

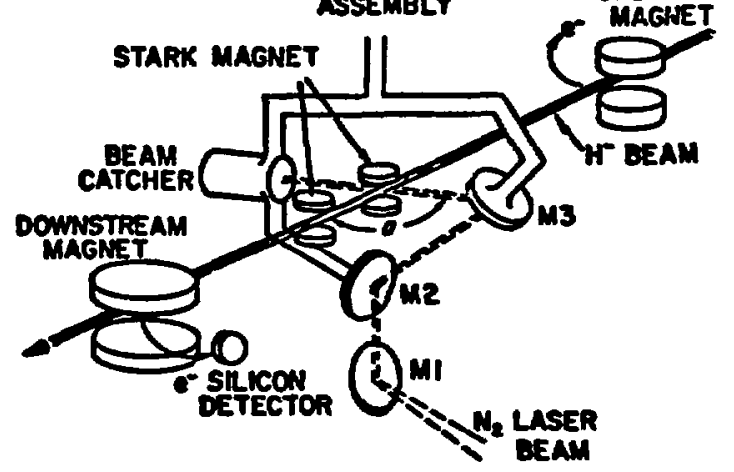

Fig. 16. Atomic physics with LAMPF: photodetachment of the negative hydrogen ion $\left(\mathrm{H}^{-}\right)$. 


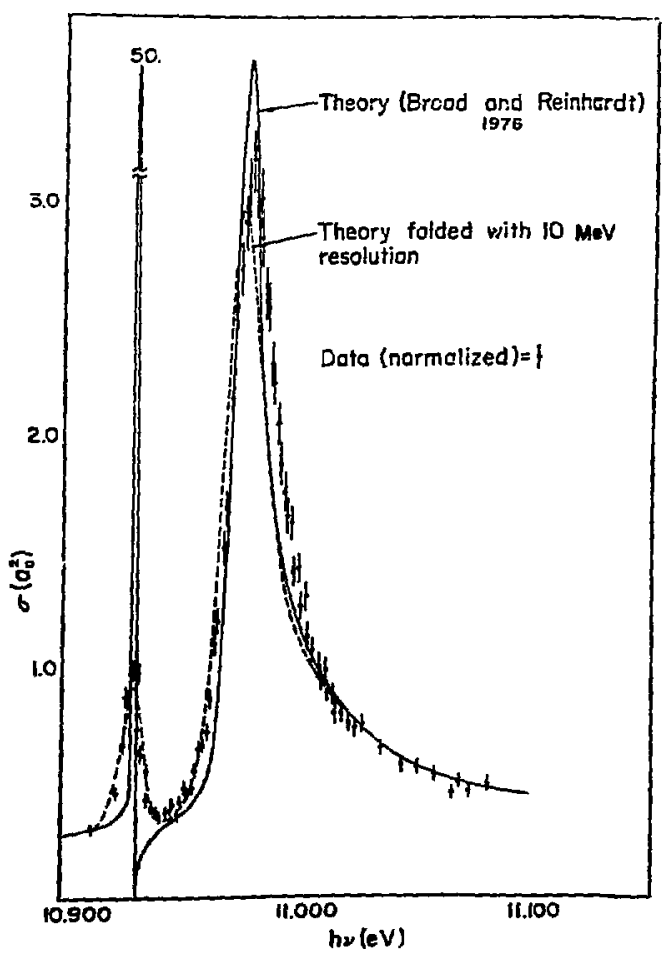

Fig. 17. Comparison of theory and experiment in photodetachment: Feshbach (L) and Shape (R) resonances.

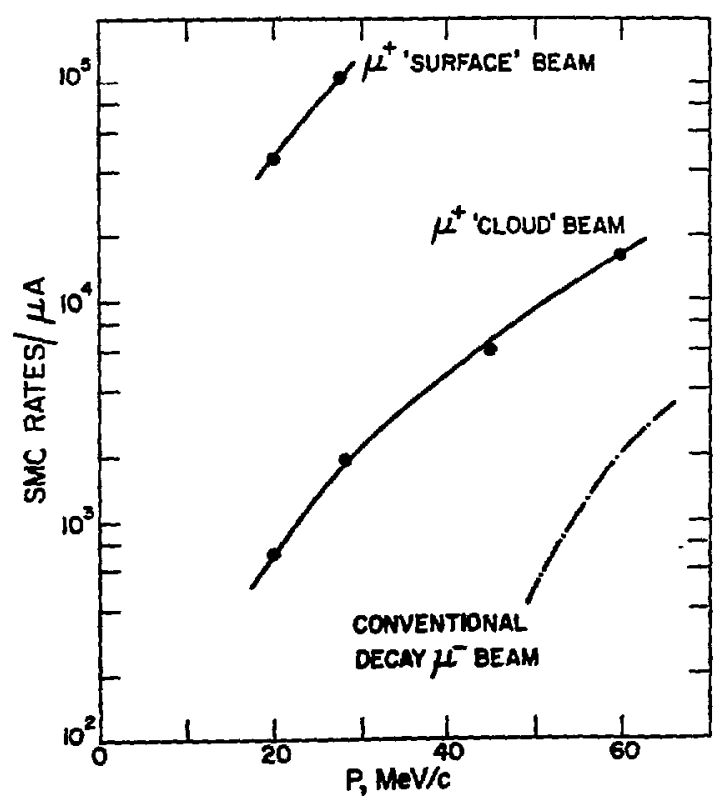

Fig. 19. Muon beams at LAMPF.

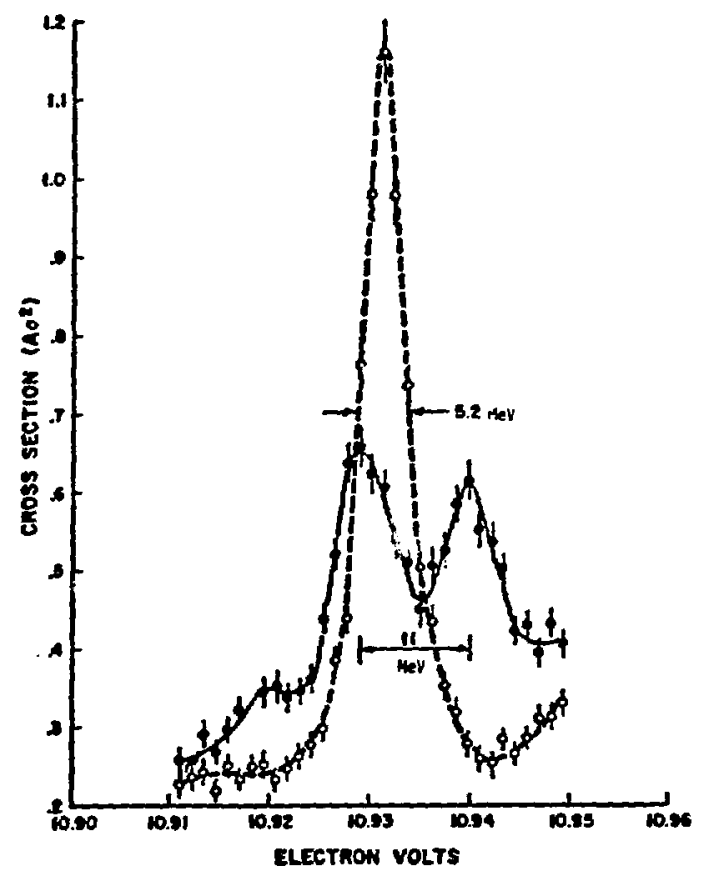

Fig. 18. Photodetachment of $\mathrm{H}^{-}$. Superposition of cross sections of Gram et al. (1978) in $n=2$ Feshbach region of $\mathrm{H}^{-}$for two different applied field values. Open circles, zero field. Closed circles, $110 \mathrm{kV} / \mathrm{cm}$ in the center of mass.

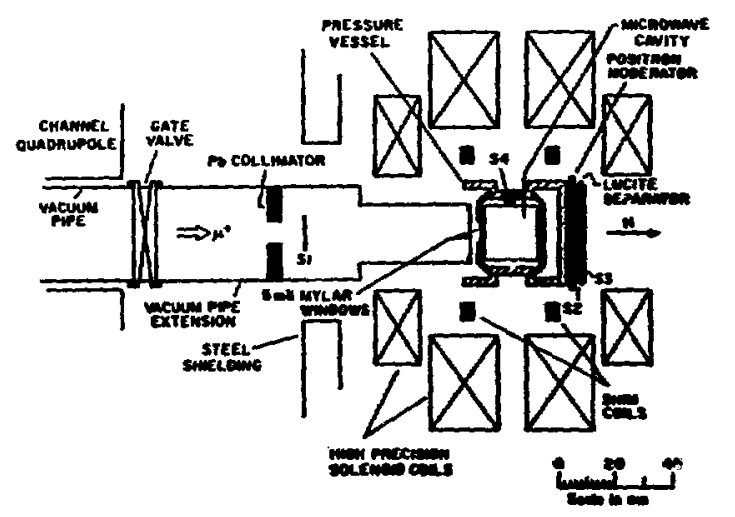

Fig. 20. Apparatus for measurenent of properties of muonitum. 


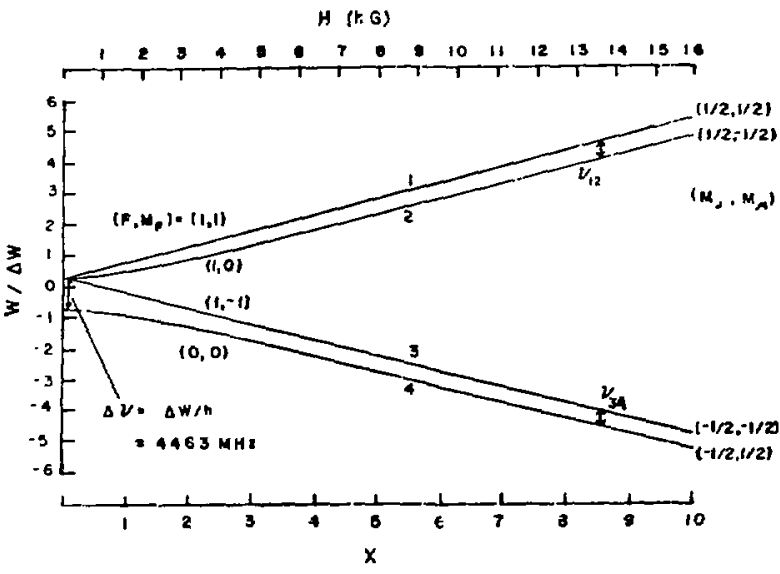

Energy level diagram for muonium in $1^{2} \mathrm{~S}_{1 / 2}$ state

$$
\begin{aligned}
& \mathcal{H}=a \vec{I}_{\mu} \cdot \vec{J}+u_{B}^{e} g_{J} \vec{j} \cdot \vec{H}-\mu_{B}^{\mu} g^{\prime} \vec{i}_{\mu} \cdot \vec{H} \\
& x=\left(g_{J} \mu_{B}^{e}+g_{\mu}^{\prime} \mu_{B}^{\mu}\right) H /(h \Delta \nu)
\end{aligned}
$$

High field results

$$
\Delta \nu=4463302.35(0.52) \mathrm{kHz}(0.12 \mathrm{ppm})
$$

$\Delta \nu$ theory $=4463318.5(6.5) \mathrm{kHz}$

$$
\mu_{\mu} / \mu_{\mathrm{p}}=3.1833403(44) \text { (1.4 ppm) }
$$

Fig. 21. The spectroscopy of muonium: hyperfine structure and magnetic moment.

\section{TWO-NUCLEON PROBLEM}

\section{Scattering Amplitudes and Phase Shifts \\ Dibaryon Resonances}

Pion Production

\section{Parify Nonconservation}

\section{Charge Symmetry; pd and nd Comparison}

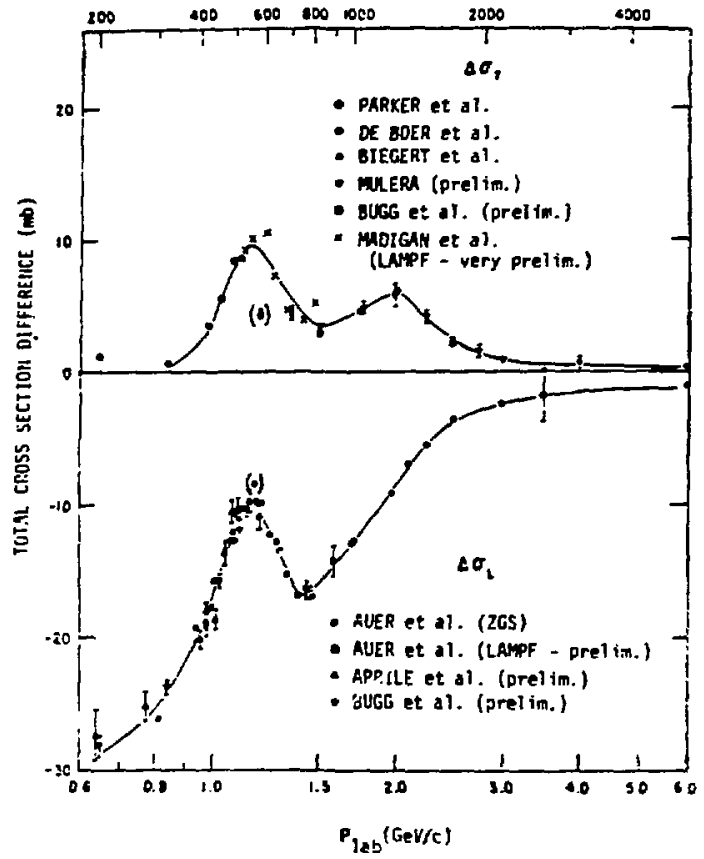

Fig. 23. $\vec{p}-\vec{p}$ scattering. Differences of total cross sections for reversals of longitudinal (L) and transverse $(T)$ polarizations vs incident momentum.

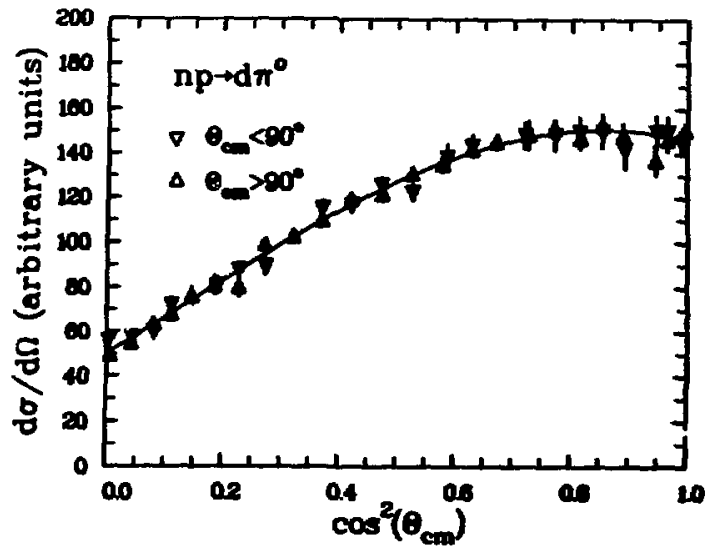

Fig. 24. Angular dependence of the differential cross section for $\mathrm{np} \rightarrow \mathrm{d \pi} \mathrm{T}^{0} . \mathrm{T}_{n}=795 \mathrm{MeV}$.

Fig. 22. The two-nucleon problem: measurements possible at LAMPF. 


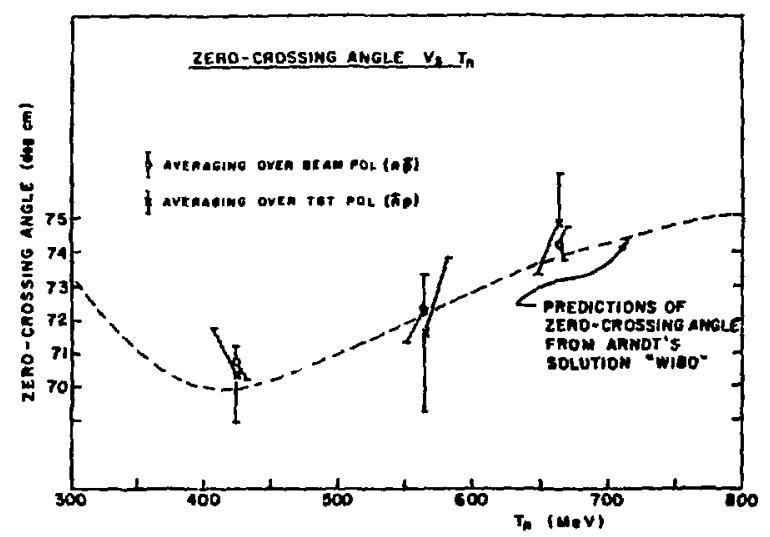

Fig. 25. Zero-crossing angle vs $T_{n}$ in $n \vec{p}$, $\overrightarrow{n p}$ scattering.

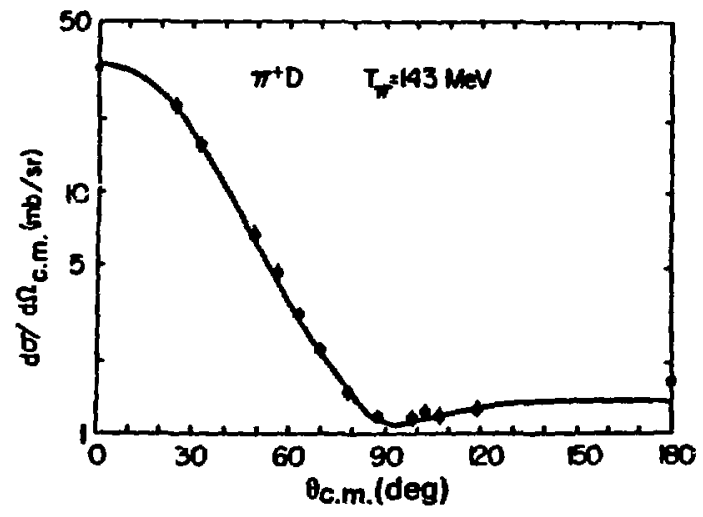

Fig. 26. Differential cross section for $\pi^{+}-D$ elastic scattering. $T_{\pi}=143 \mathrm{MeV}$.

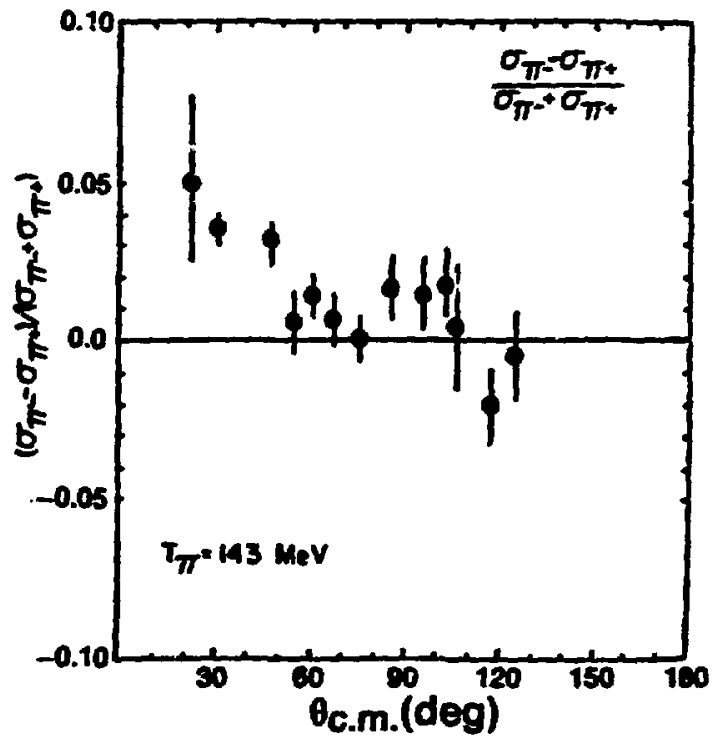

Fig. 27. Charge dependence of differential cross section for $\pi^{ \pm}-D$ scattering vs angle. $T_{\pi}=$ $143 \mathrm{MeV}$.

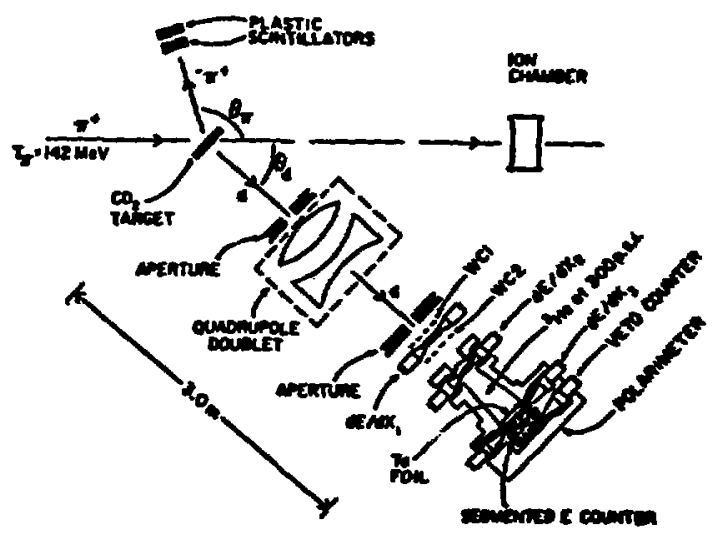

Fig. 28. Experimental arrangement for measuring the polarization of the recoil deuteron in $\pi-D$ scattering. 


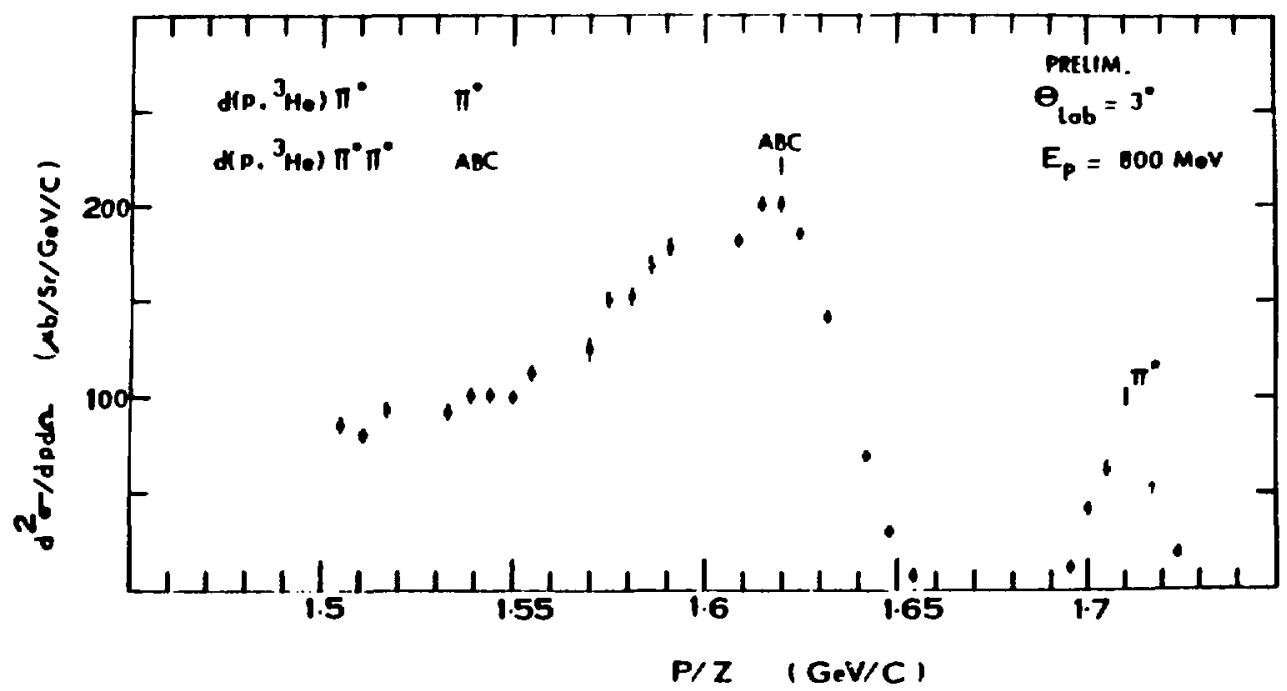

Fig. 29. Double differential cross sections for $p+d \rightarrow^{3} \mathrm{He}+m$ (missing mass) reactions vs momentum $(P / Z)$.

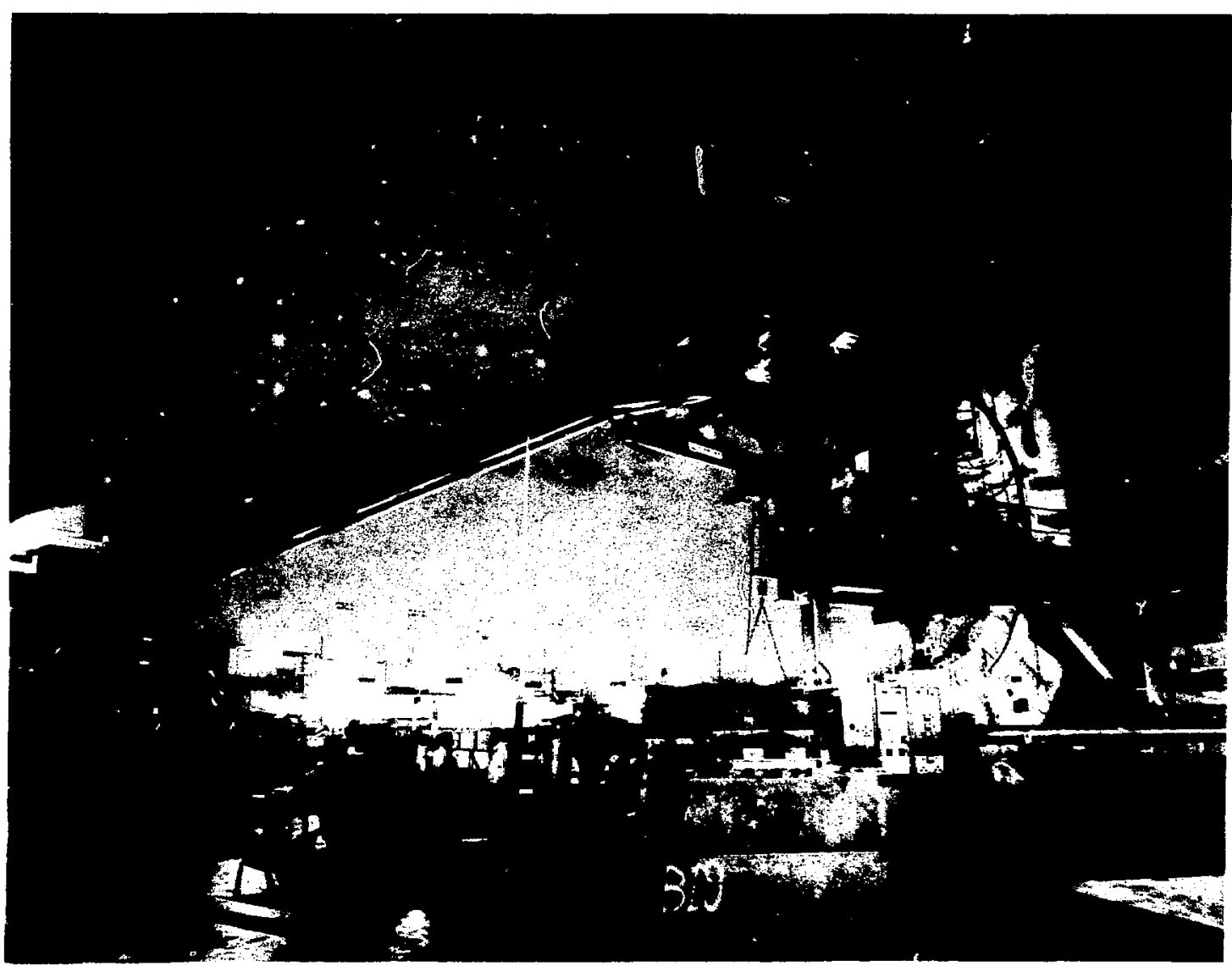

Fig. 30. High-Resolution Spectrometer (HRS). 


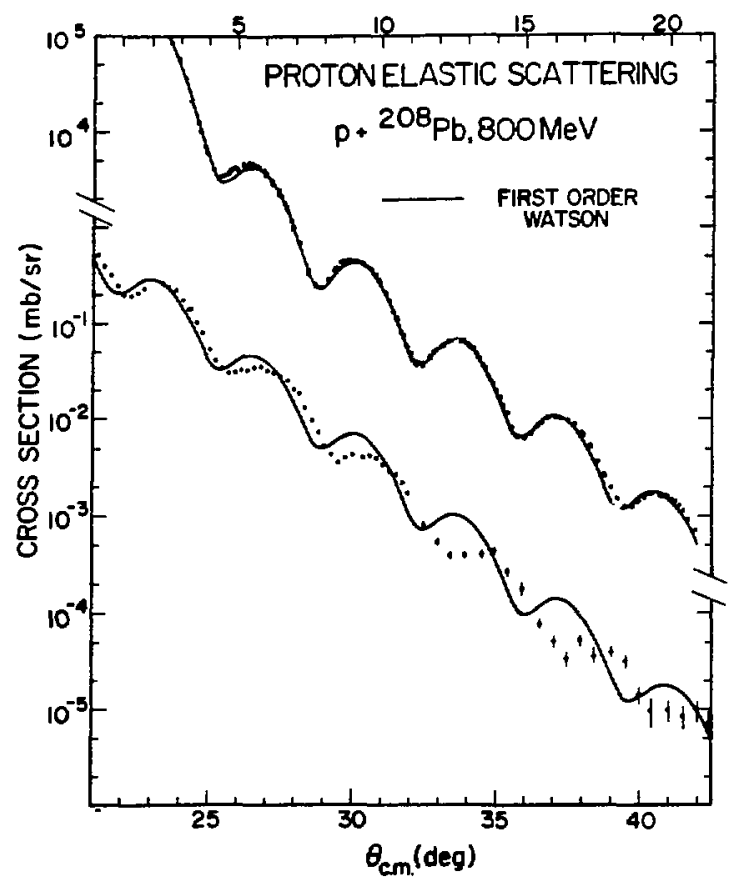

Fig. 31. p-nucleus data from HRS: differential cross section for $\mathrm{p}-208 \mathrm{pb}$ elastic scattering. $T_{p}=800 \mathrm{MeV}$.

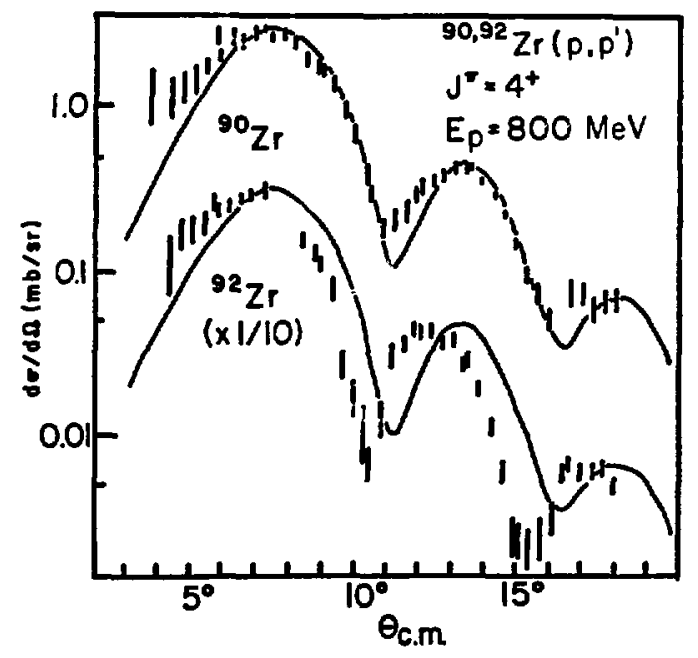

Fig. 33. Differential cross section for $90,92 \mathrm{Zr}\left(p, p^{\prime}\right)$ vs angle. $T_{p}=800 \mathrm{MeV}$.

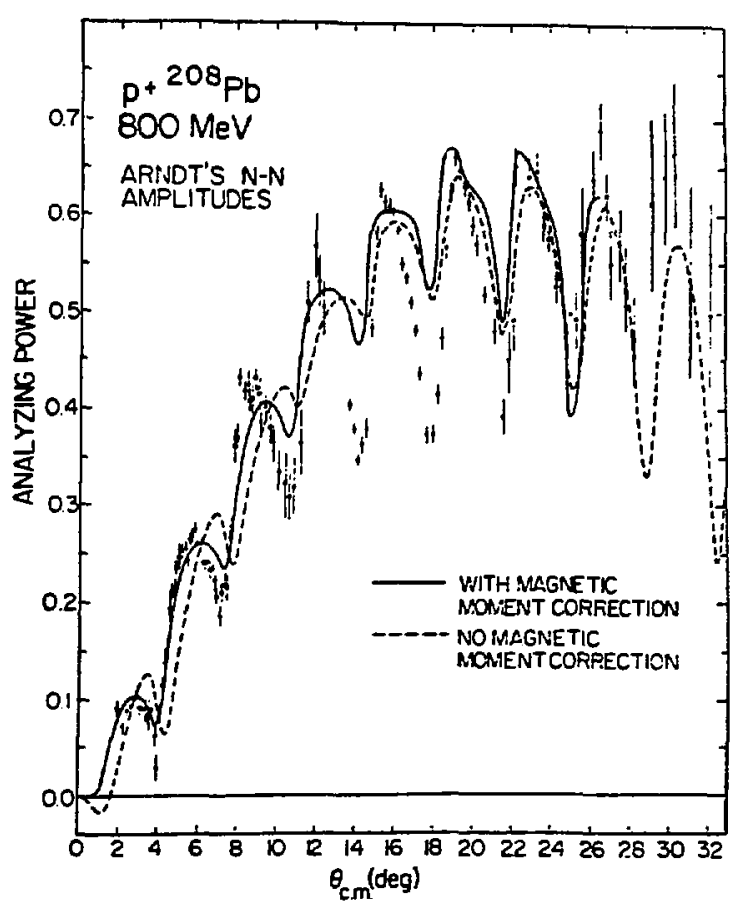

Fig. 32. Analyzing power in $\mathrm{p}-{ }^{208} \mathrm{pb}$ scattering compared to KMT predictions.

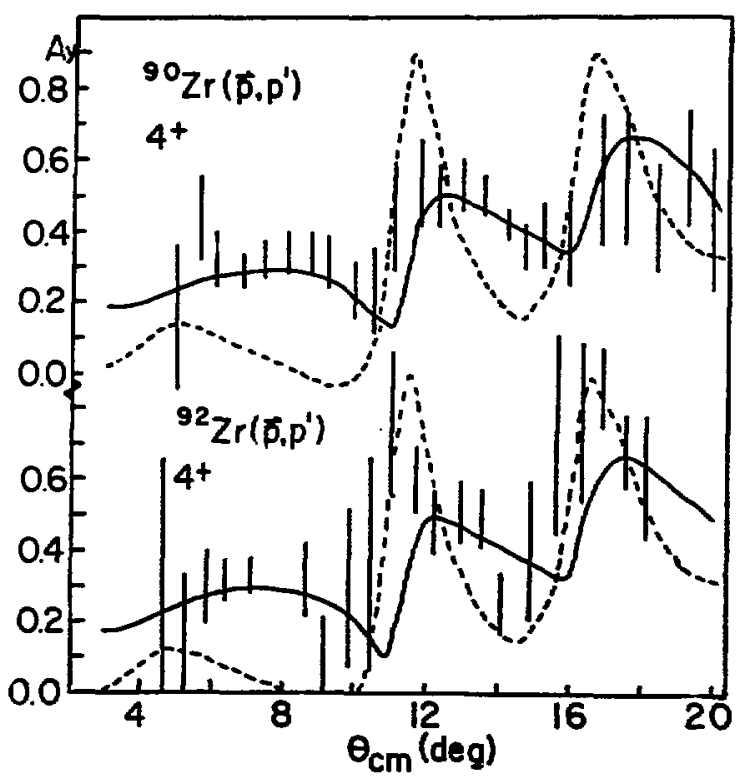

Fig. 34. $A_{y}(=\sigma t-\sigma+/ \sigma \uparrow+\sigma t)$, the polarizafjon asymetry vs angle in $\mathrm{p}-12 \mathrm{C}$ elastic scattering. 


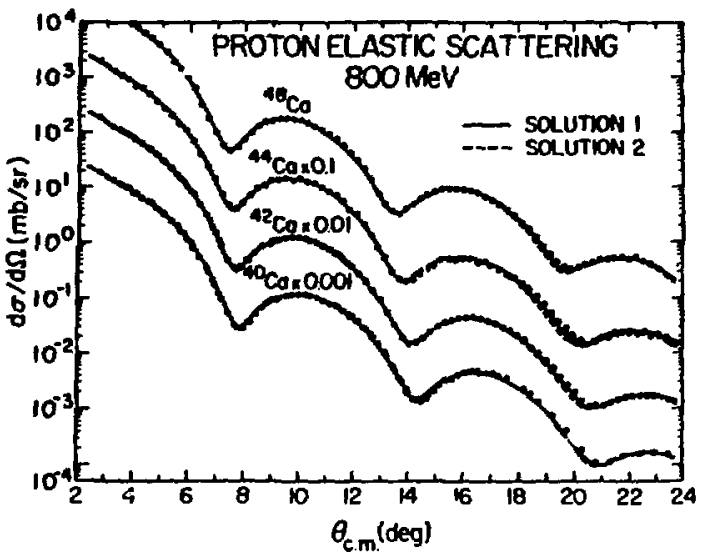

Fig. 35. Differentiai cross section for elastic scattering of protons from calcium isotopes vs angle. $T_{p}=800 \mathrm{MeV}$.

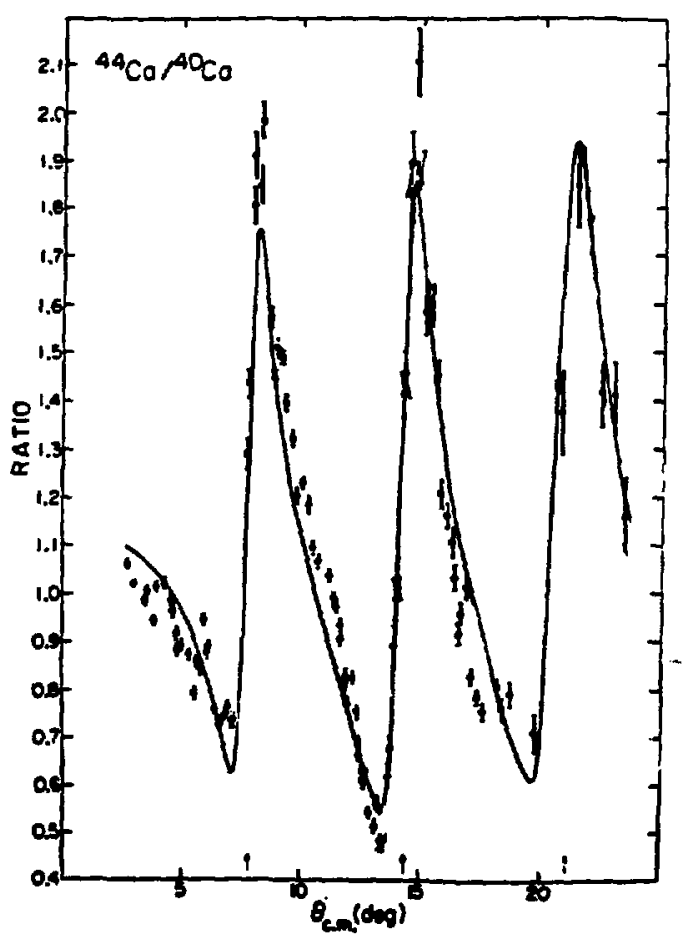

Fig. 36. Ratio of differential cross sections for elastic scattering of protons from $44 \mathrm{Ca}$ and $40 \mathrm{Ca}$ vs angle. $\mathrm{T}_{\mathrm{p}}=800 \mathrm{MeV}$. Solid line is a theoretical curve.

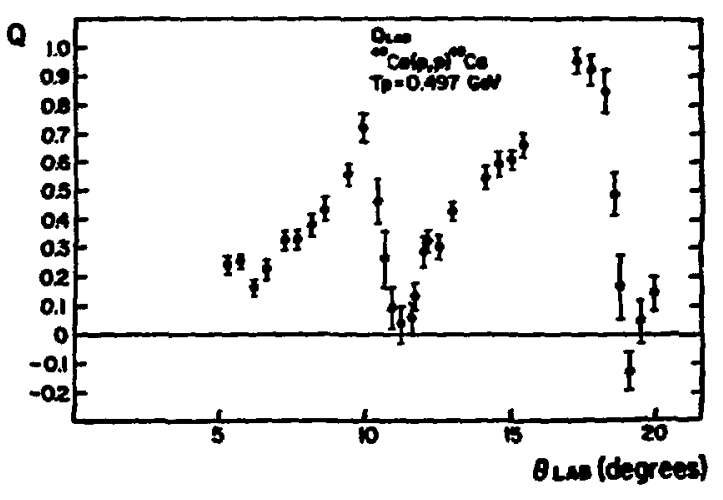

Fig. 37. Measurement of the spin-rotation parameter $Q$ in the scattering of $0.5-\mathrm{GeV}$ polarized protons from $40 \mathrm{Ca}$. (See text for definition of Q.)
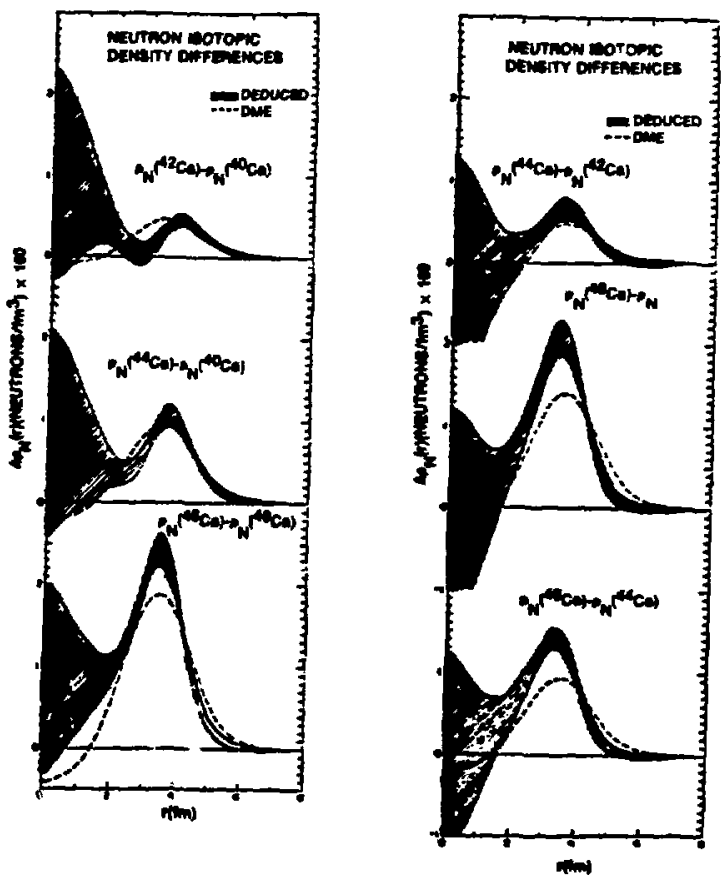

Fig. 38. Calculated neutron-density distribution differences for the calcium isotopes. Density Matrix Expansion (DME) is a Hartree-Fock prediction. 

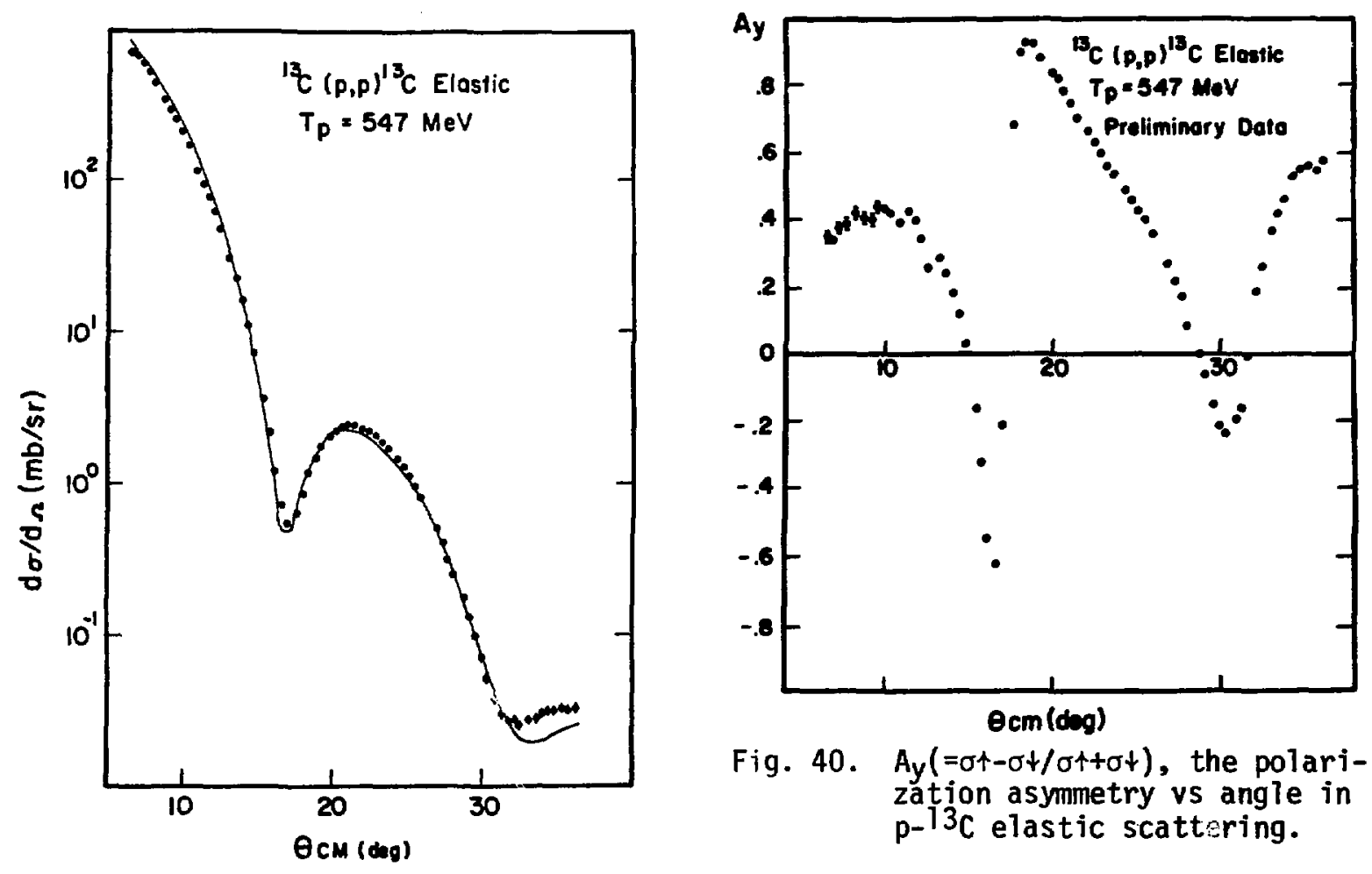

Fig. 39. Differential cross section for $13 \mathrm{C}(\mathrm{p}, \mathrm{p}) 73 \mathrm{C}$ at $\mathrm{T}_{\mathrm{p}}=547$ MeV.

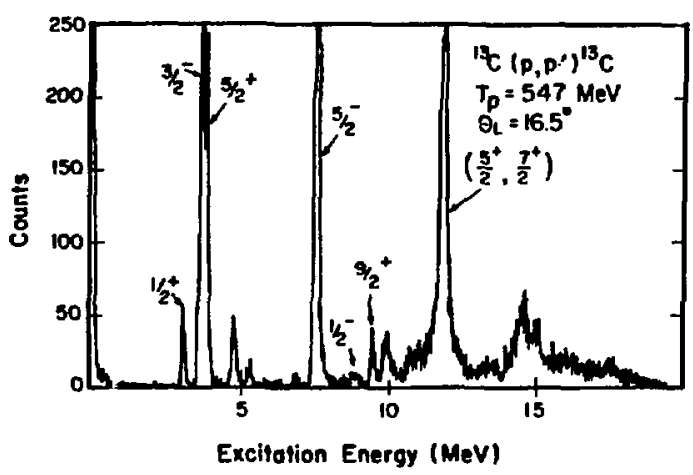

Fig. 47. $p-13 c$ inelastic scattering vs excitation energy.

Fig. 42. Differential cross section for $\mathrm{p}-{ }^{3} \mathrm{C}$ inelastic scattering vs angle. $T_{p}=547 \mathrm{MeV}$.

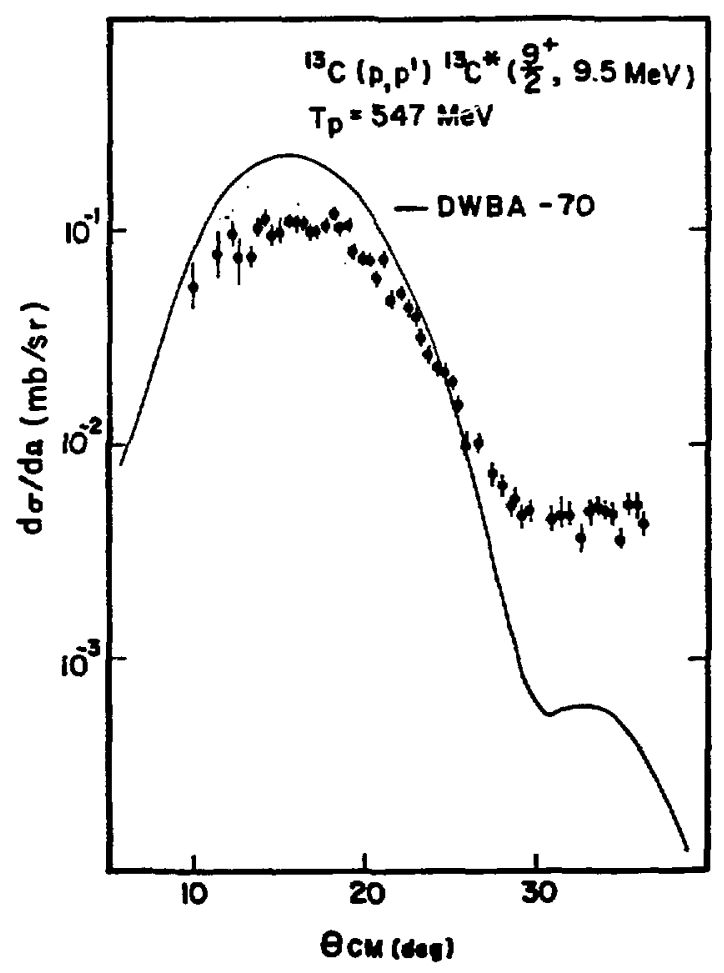




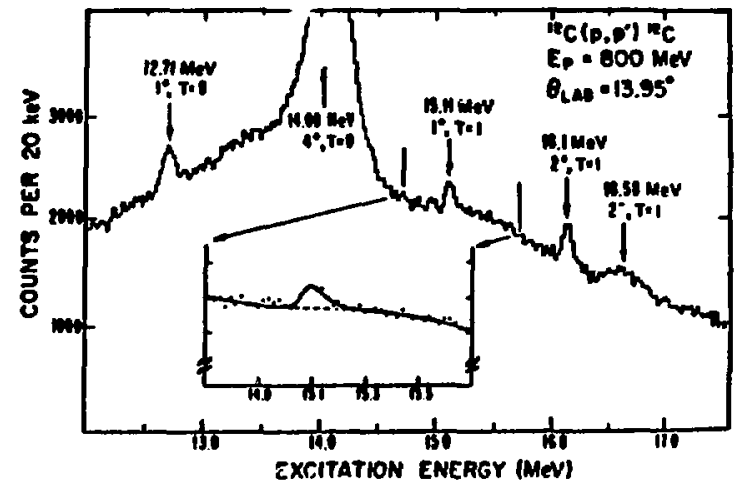

Fig. 43 Test for critical opalescence (see text), inelastic proton scattering from ${ }^{12} \mathrm{C}$ vs excitation energy. $T_{p}=800 \mathrm{MeV}$.

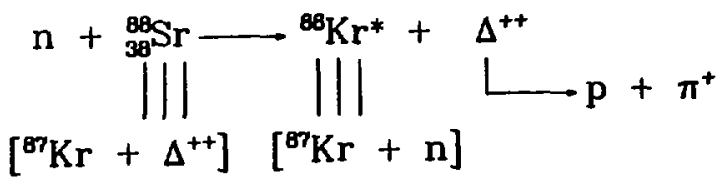

Fig. 45. Search for $\Delta^{++}(1232)$ in a complex nucleus by a radiochersical technique.

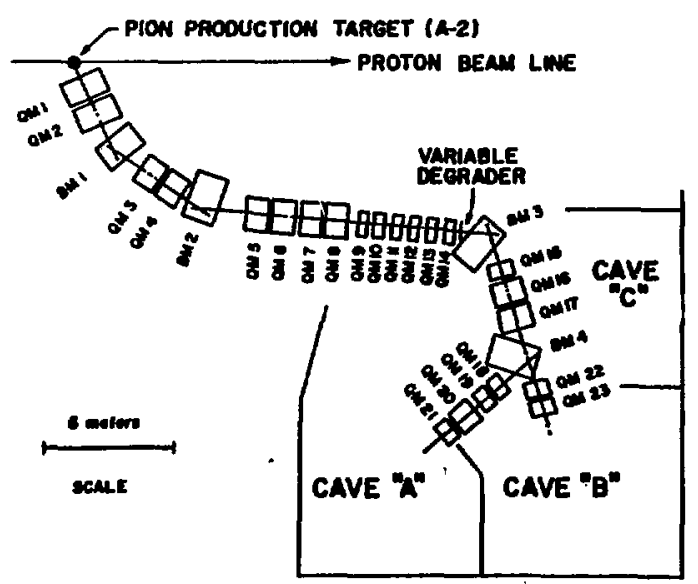

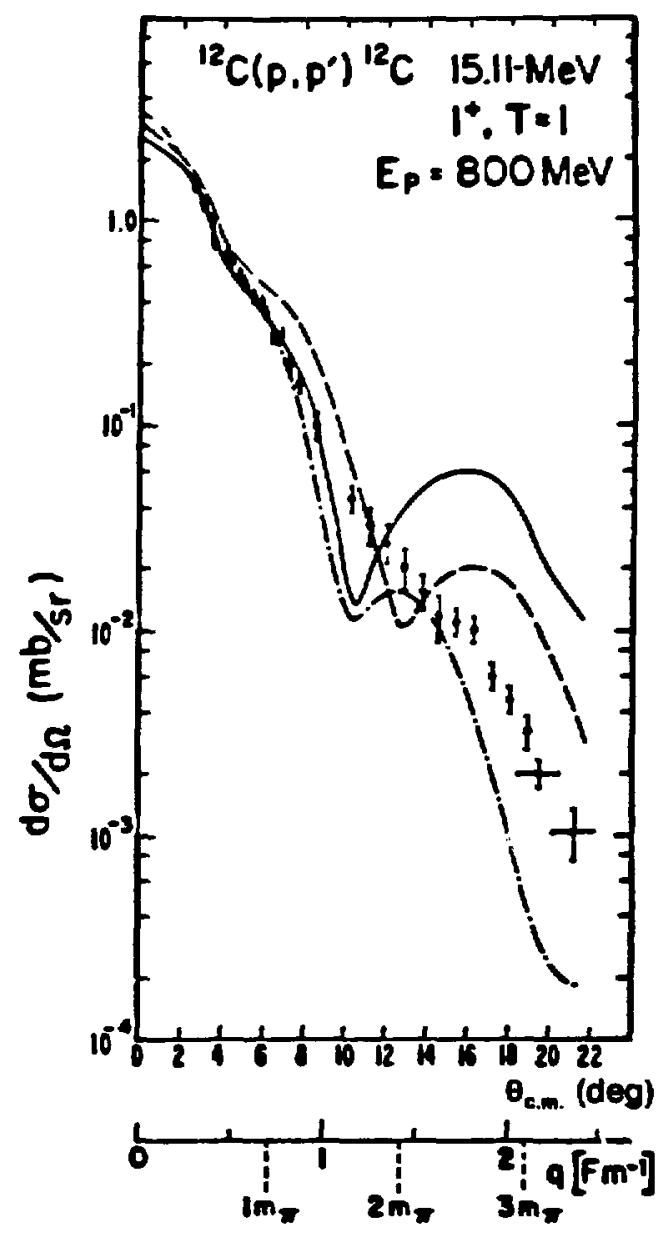

Fig. 44. Test for critical opalescence (see text), differentjal cross section for $\mathrm{p}-12 \mathrm{C}$ inelastic scattering vs angle. $\mathrm{T}_{\mathrm{p}}=800 \mathrm{MeV}$.

Fig, 46. Schematic of Stopped Muon Channel (SMC). 


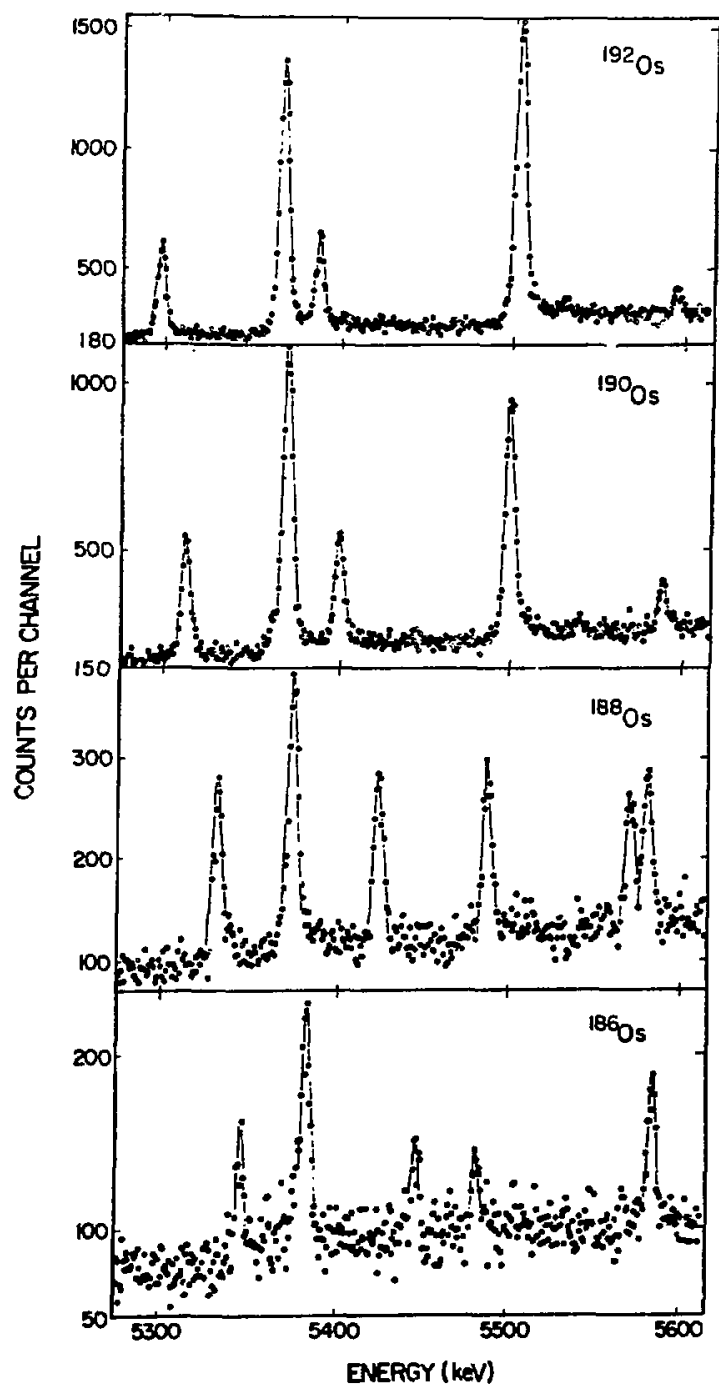

Fig. 47. Muonic x-ray spectra of osmium isotopes in the region of the $2 p-1 s$ transitions.

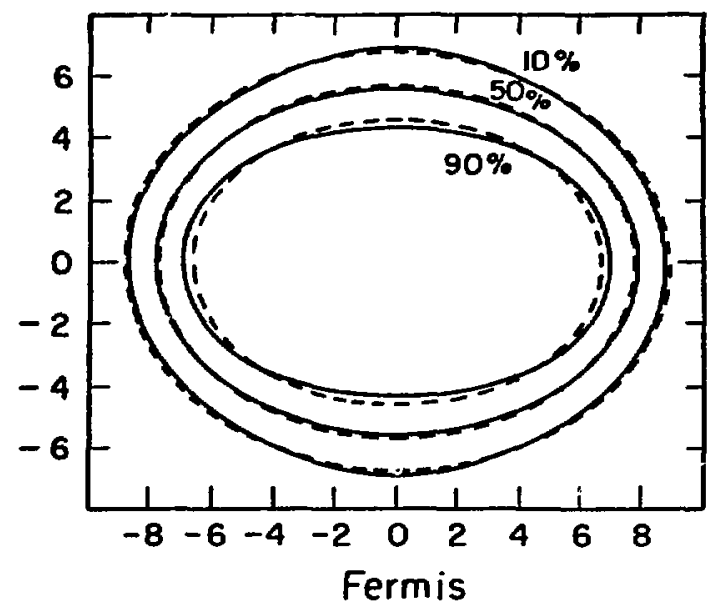

Fig. 48. Charge distribution $t$. $165 \mathrm{Ho}$. 


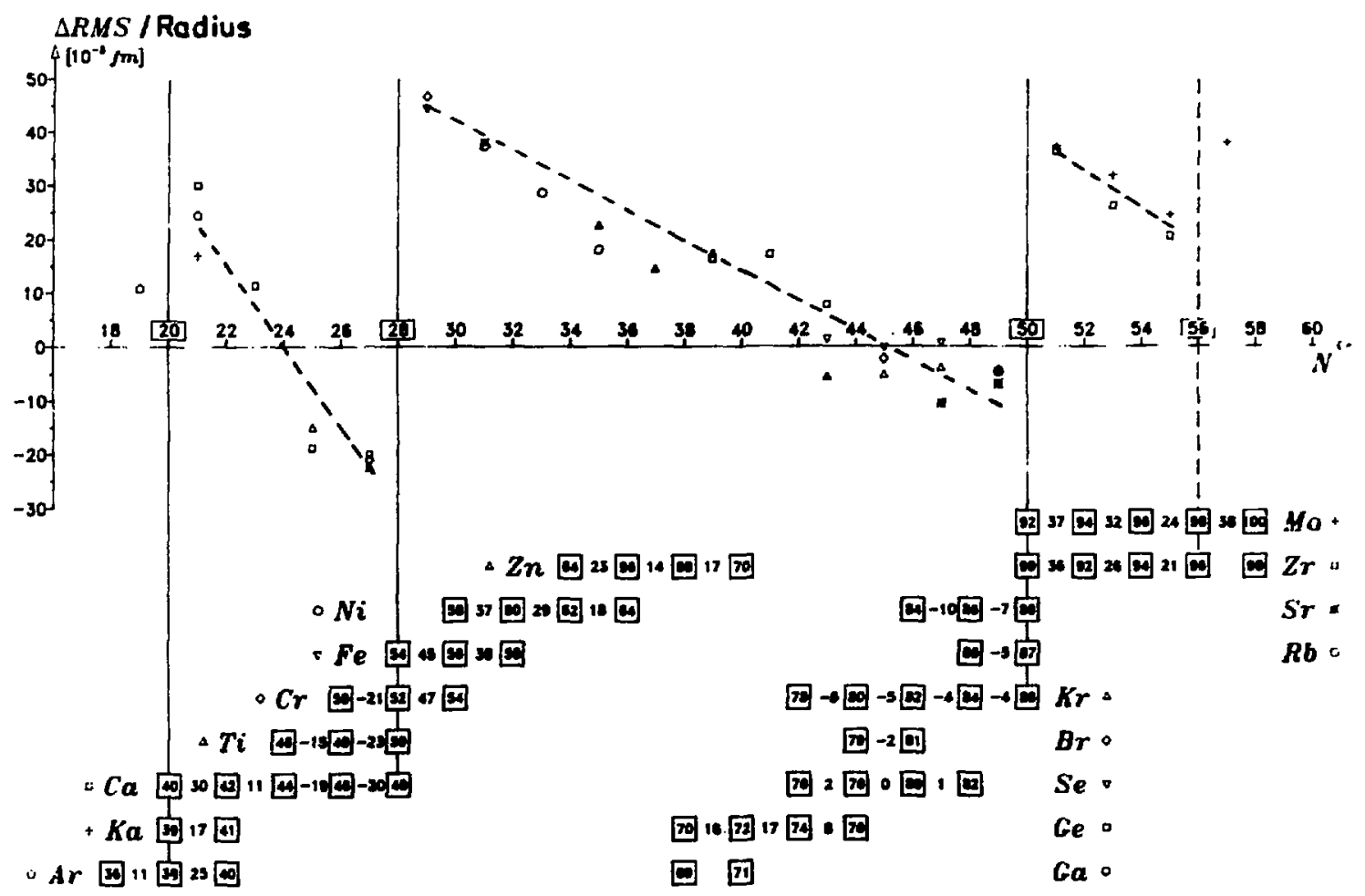

Fig. 49. Differences in nuclear charge radii for various nuclei upon addition of two nuclei.

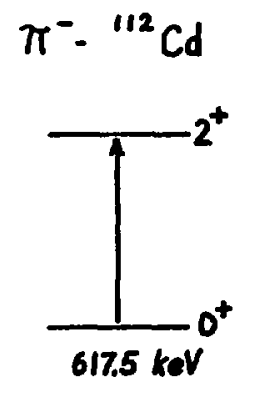

NUCLEAR

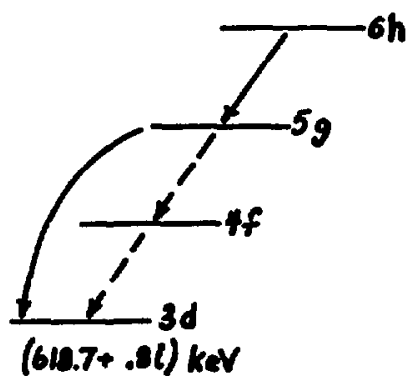

ATOMIC

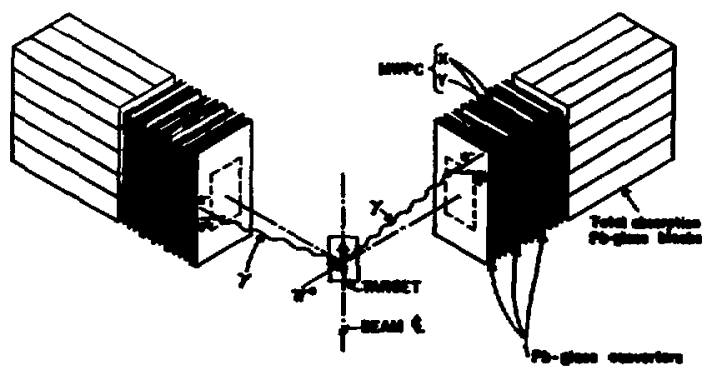

Fig. 50. Levels involved in the nuclear reşonance effect in pionic

Fig. 51. $\pi^{0}$ spectrometer. 


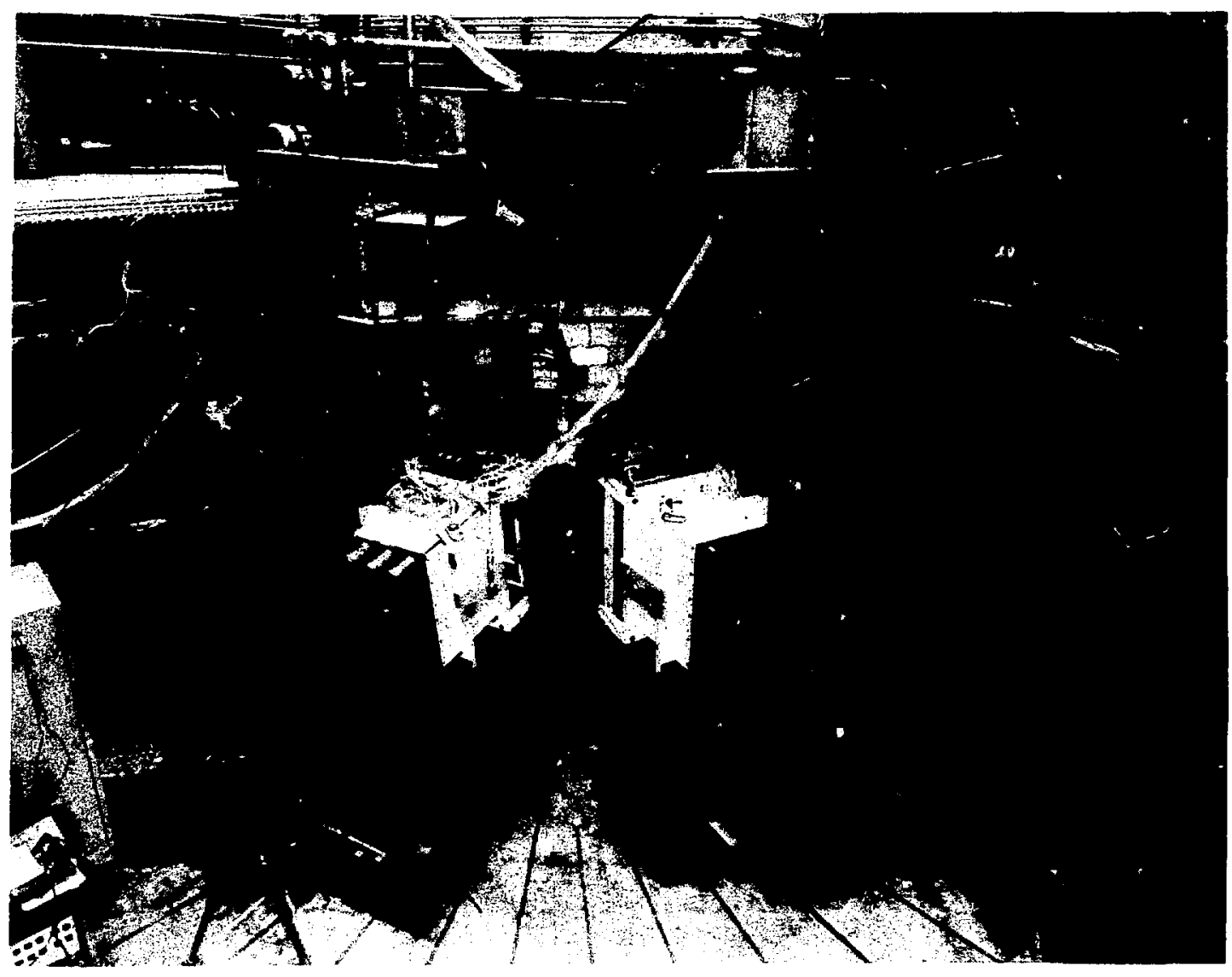

Fig. 52. $\pi^{0}$ spectrometer. 


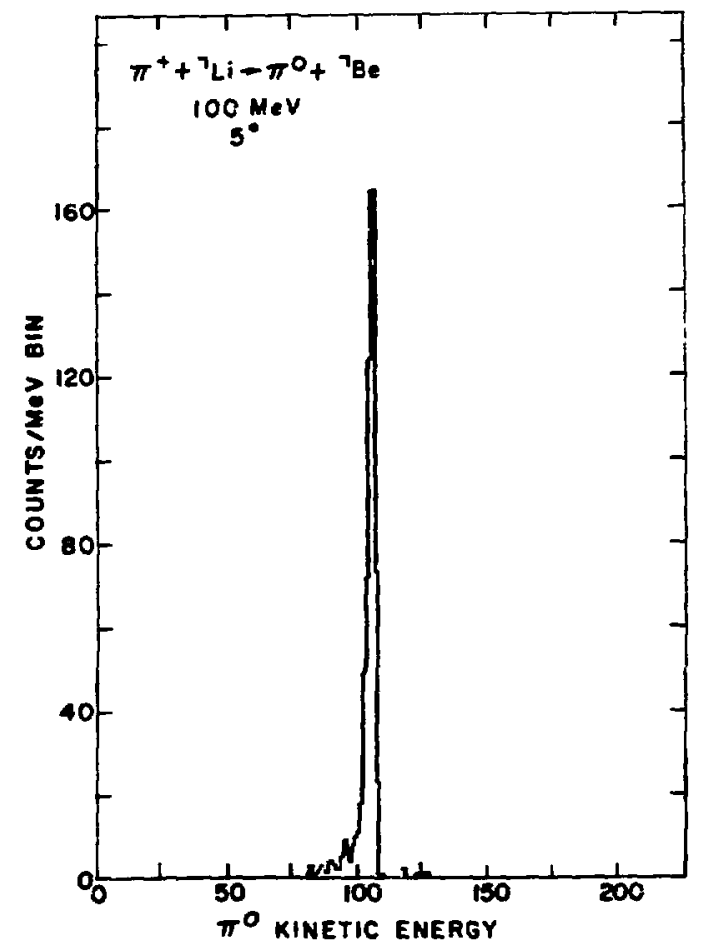

Fig. 53. Fundamental $\pi^{+} g \rightarrow \pi^{0}$ reaction observed in a $\mathrm{Z}_{\mathrm{Li}}$ target. $\mathrm{T}_{\pi^{+}}=100 \mathrm{MeV}$.

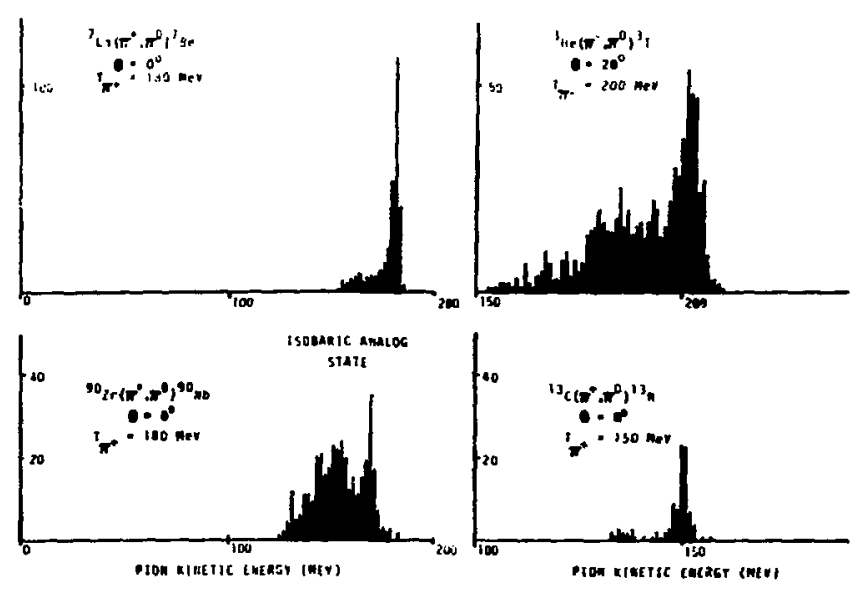

Fig. 54. Isobaric analog transitions observed with $\pi^{0}$ spectrometer. 


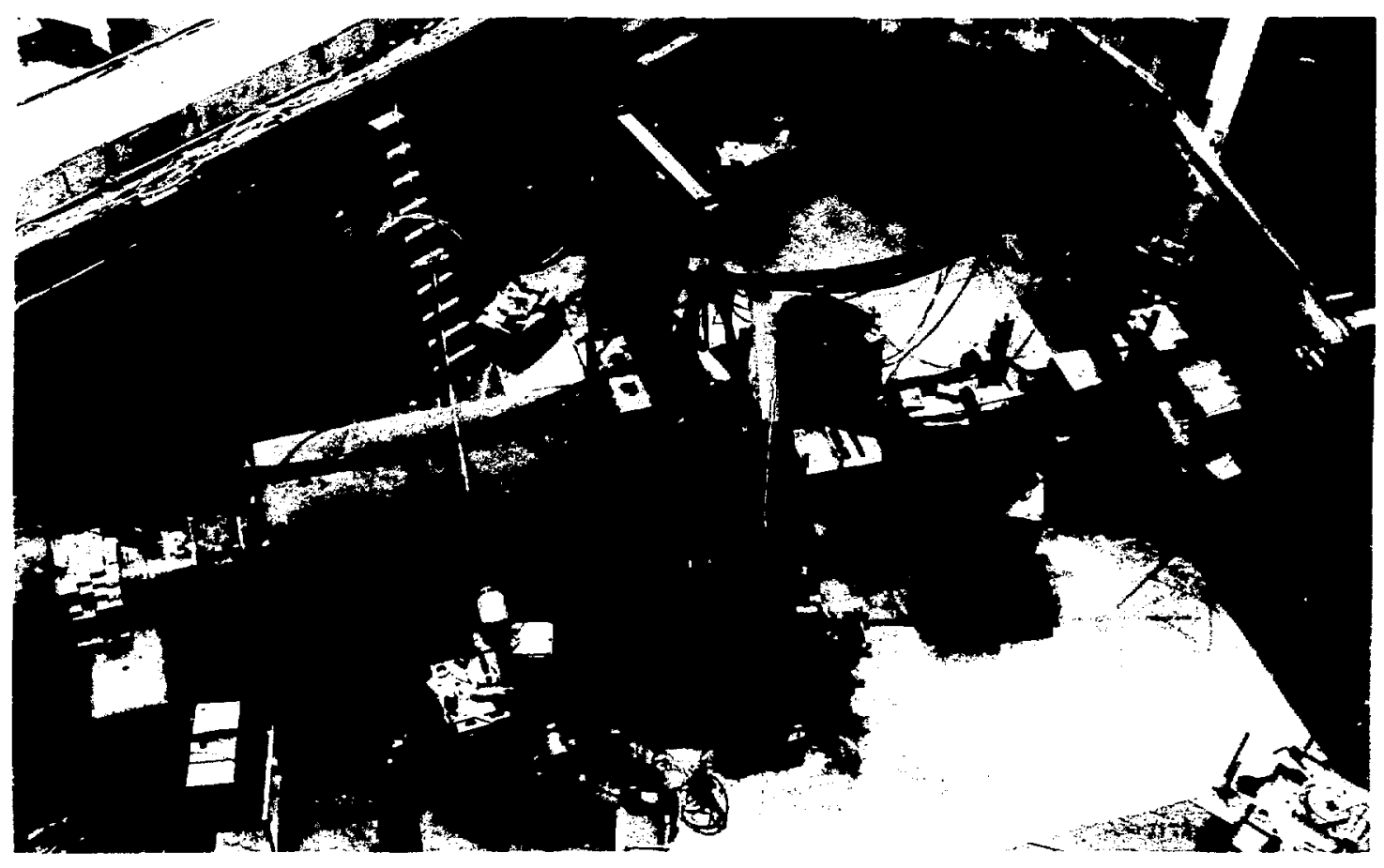

Fig. 55. Apparatus for detection of the rare decay $\pi^{ \pm} \rightarrow \pi^{0}+e^{ \pm}+\nu$. Pions of energy $400-\mathrm{MeV}$ decay with in the large evacuated cylinder and are detected by the $\pi^{0}$ spectrometer. 


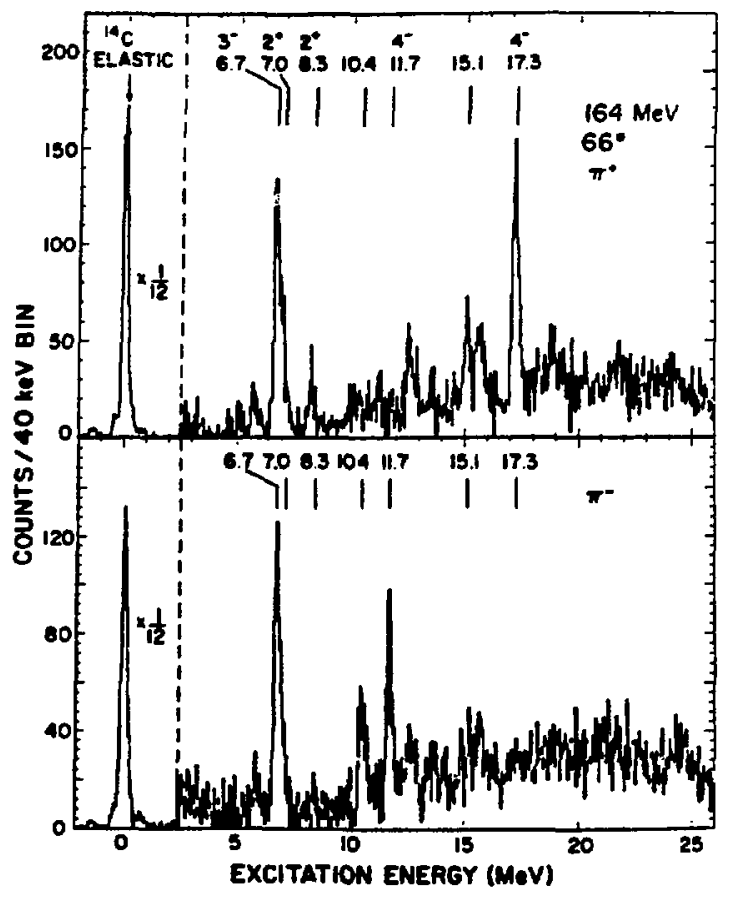

Fig. 56. Inelastic $\pi^{ \pm}$scattering on $14 \mathrm{C}$ vs excitation energy.

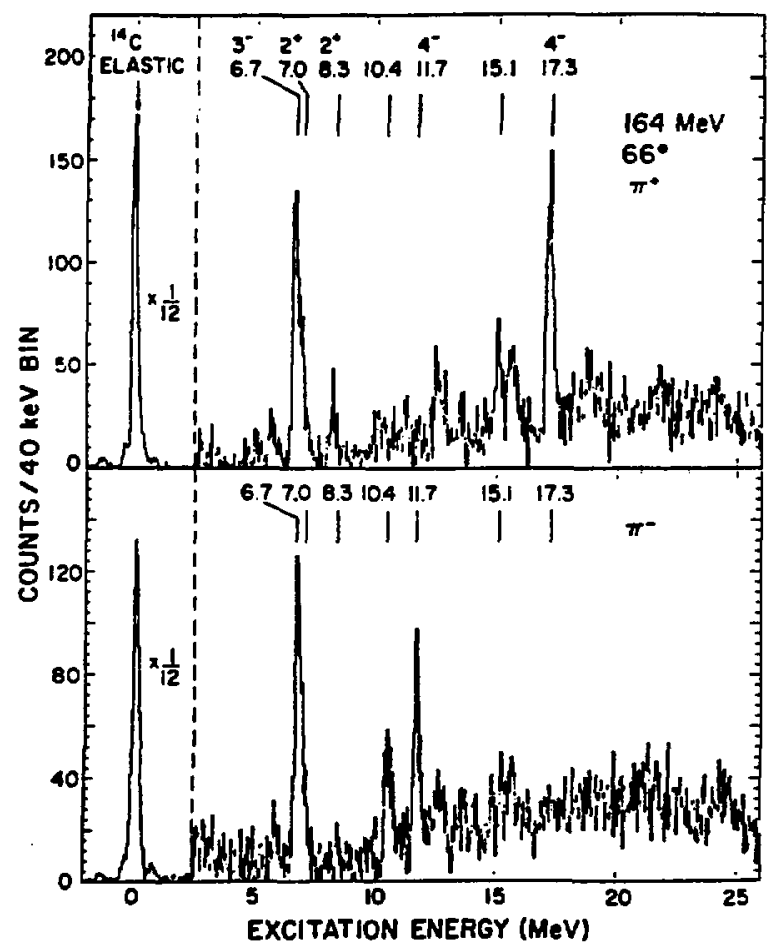

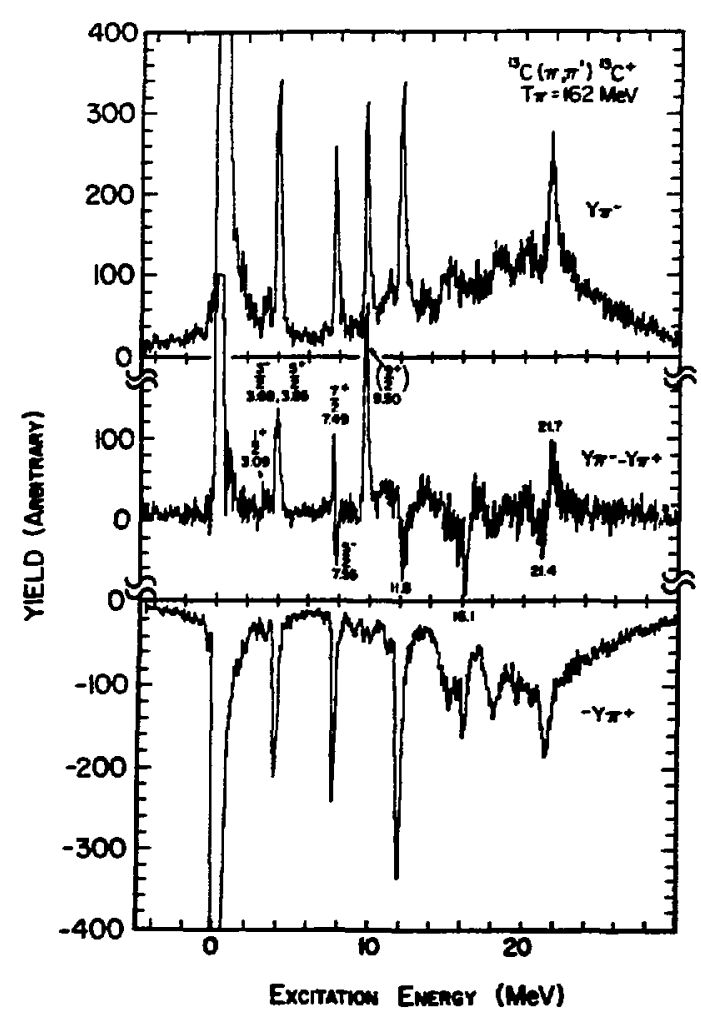

Fig. 57. Inelastic $\pi^{ \pm}$scattering on ${ }^{13} \mathrm{C}$ vs excitation energy.

Fig. 58. Inelastic $\pi^{ \pm}$scattering on
$1_{4} \mathrm{C}$ vs excitation energy. 
EXPERIMENT \#25 - DOUBLE CHARGE EXCHANGE

(los Alamos, Univ. South Carolina, anl)

THE $\left(\pi^{*}, \pi^{\circ}\right)$ REACTION ON A NUCLEUS CHANGES TWO NEUTRONS NTO TWO PROTONS:

$$
Z^{A} N\left(\pi^{*}, \pi^{-}\right) Z+2^{A} N-2
$$

THE CROSS-SECTION $d \sigma / d \Omega\left(0^{\circ}\right)$ WAS OBSERVED BY USING A PION CHANNEL AS BOTH A $\pi^{*}$ CHANNEL (IST HALF) AND AT" SPECTROMETER (2 ${ }^{\text {NO }}$ HALF):
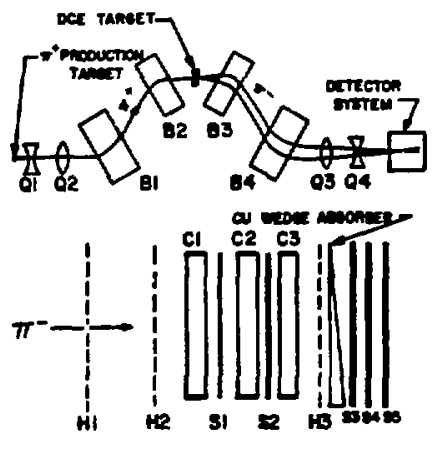

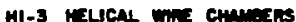

Cl-s PLASTIC EESEMKOV courtens

H -5 ConTLLLATON couwtens

DETALS OF DETECTOR SYSTEM

Fig. 59. Pion double charge exchange (DCX) at the Low Energy Pion Channel (LEP).

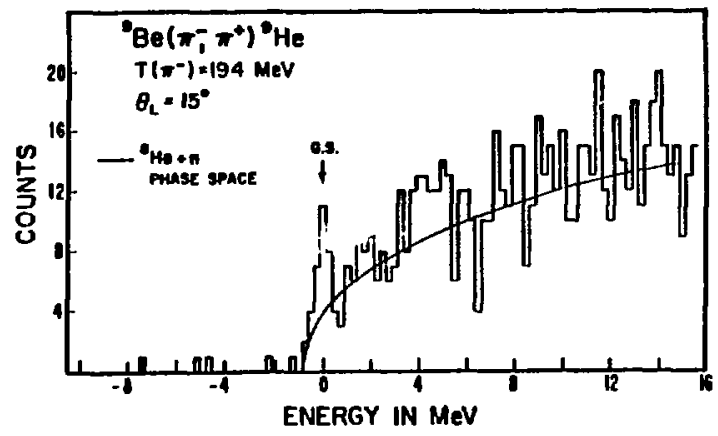

Fig. 67. Pion double charge exchange on ${ }^{9} \mathrm{Be}$ vs $\mathrm{Q}$ value.

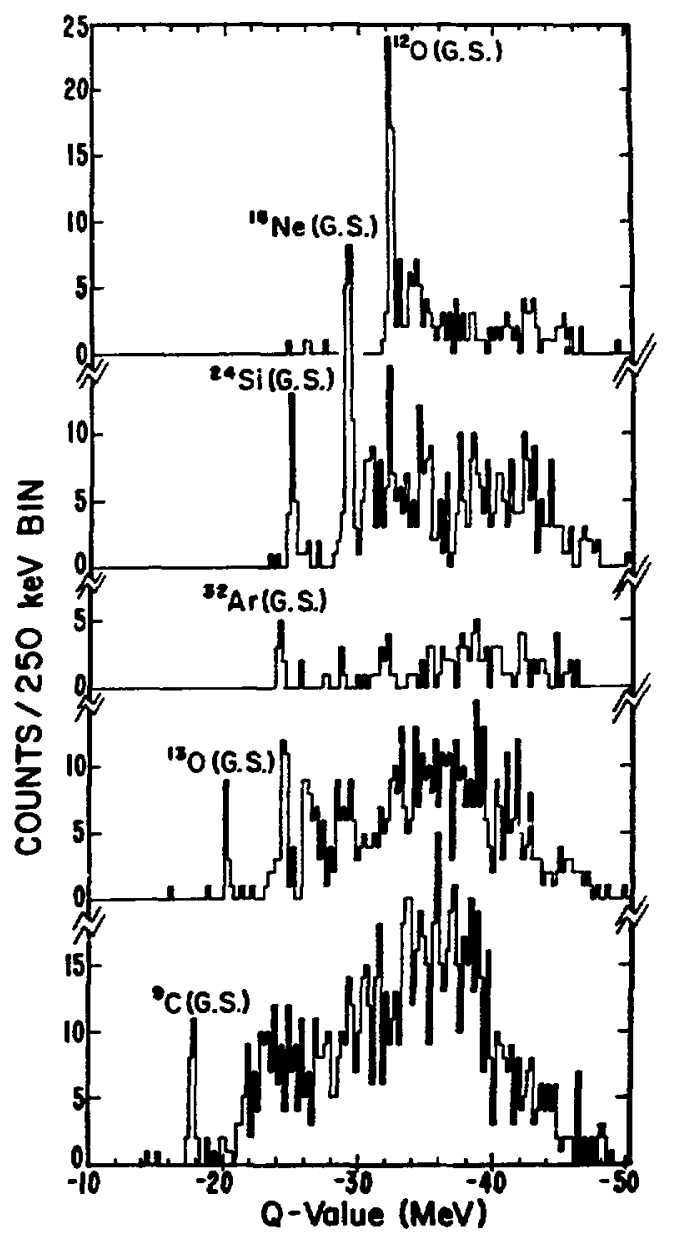

Fig. 60. Pion double charge exchange on various nuclei vs $Q$ value.

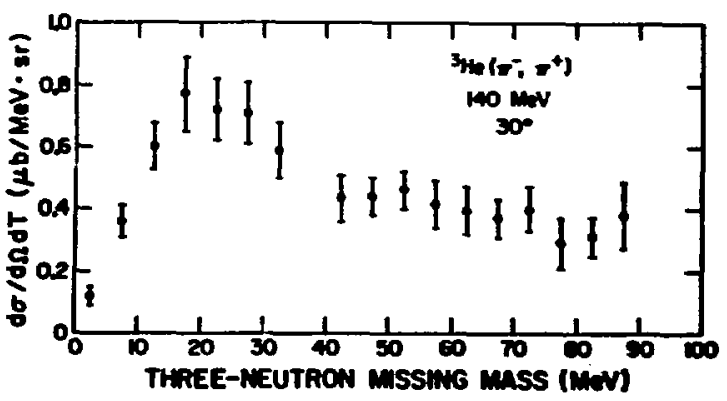

Fig. 62. Pion double charge exchange on $3_{\mathrm{He}}$ resulting in the three-neutron system. 


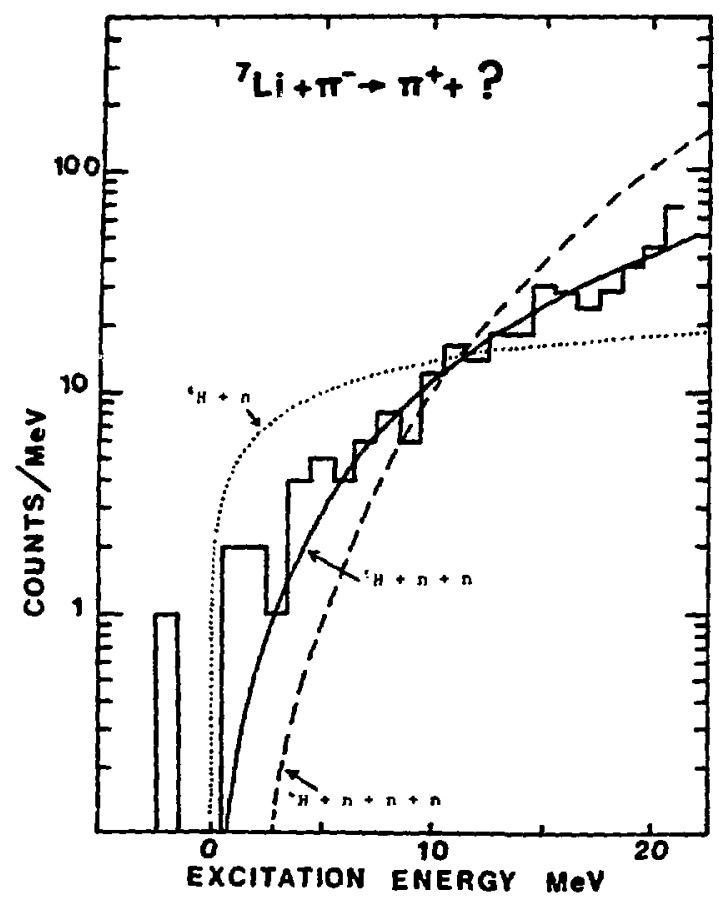

Fig. 63. Pion double charge exchange on $7_{L i}:$ various final states

$\pi^{ \pm}$-Nucleus Interactions

- Elastic Scattering

- Inelastic Scattering

- Single Charge Exchange

- Double Charge Exchange

Muonic Atoms

-Charge Distribution

$\mu^{ \pm}$-Nucleus Interactions

Proton-Nucleus Interactions

\section{Matter Distributions}

Fig. 64. Methods of investigating nuclear structure.
Neutrino Effects:

- Lepton Conservation

- Neutrino Oscillations I

- $\gamma$-e- Scattering

- Neutrino Oscillations II

Pare Decay of Muon:

$$
\begin{aligned}
& \mu \rightarrow \text { er I } \\
& \mu \rightarrow \text { er II } \\
& \mu \rightarrow \text { 3e; } \mu \rightarrow \text { err }
\end{aligned}
$$

$\mu^{+}$Decay Spectrum

Fig. 65. Weak interactions and unification theories.

EXperiment \#31 - A Test of MUON Conservation

Yale, los alamos, irc-canada, Saclay, Sim

$\mu \rightarrow e^{+}+\nu_{e}+\overline{\nu_{\mu}} \quad$ additive or

$\mu^{+} \rightarrow e^{+}+\bar{\nu}_{e}+\nu_{\mu} \quad$ multiplicative (both) 


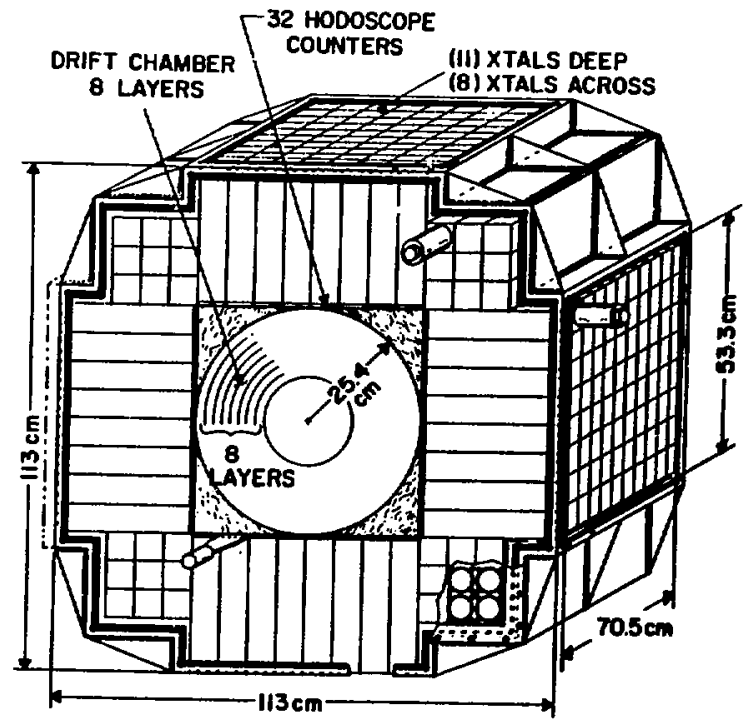

Fig. 67. The crystal box, an apparatus for observation of rare decays of the muon.

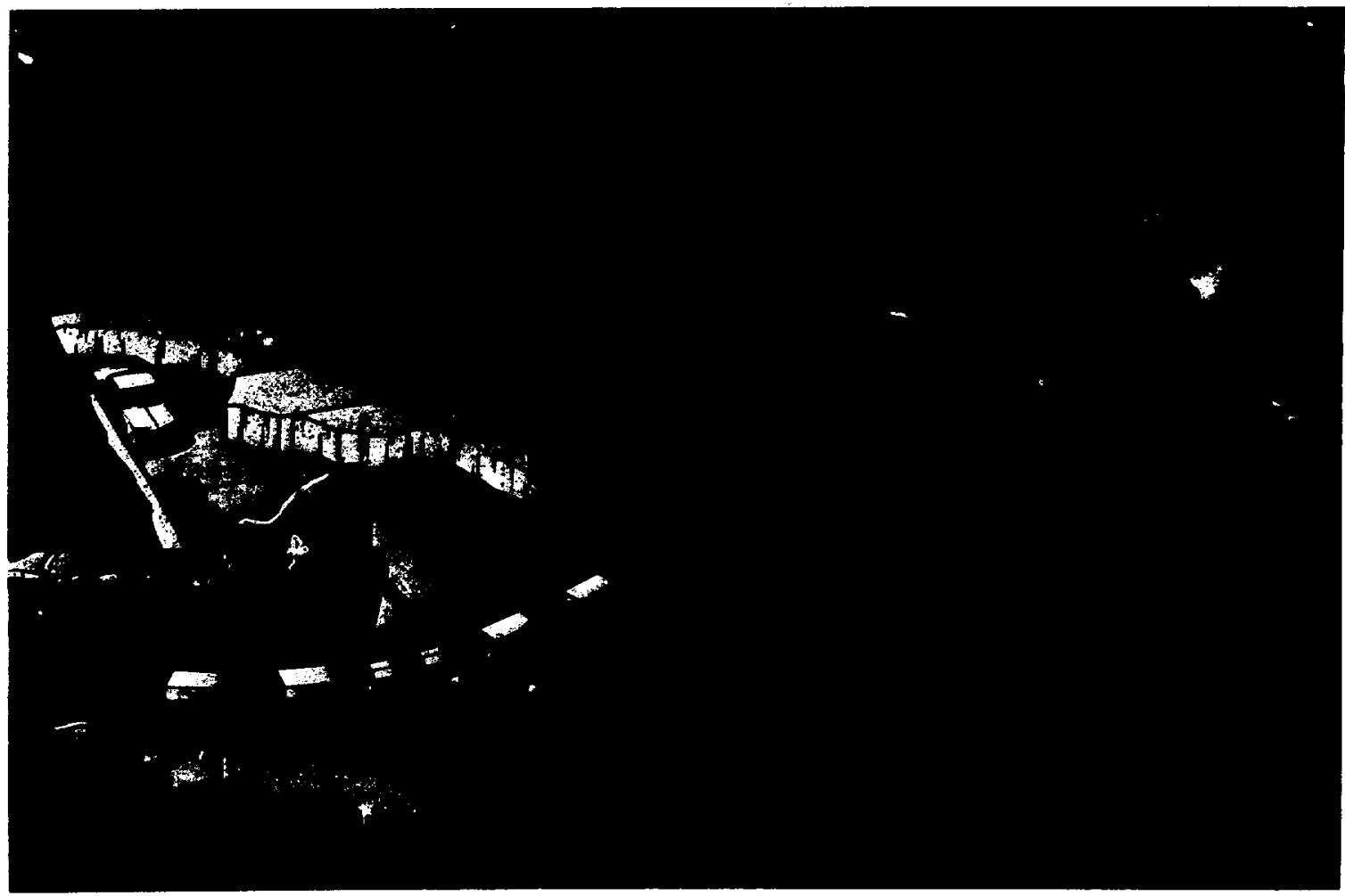

Fig. 68. Neutrino facility: proposed neutrino production tunnel (1982). 


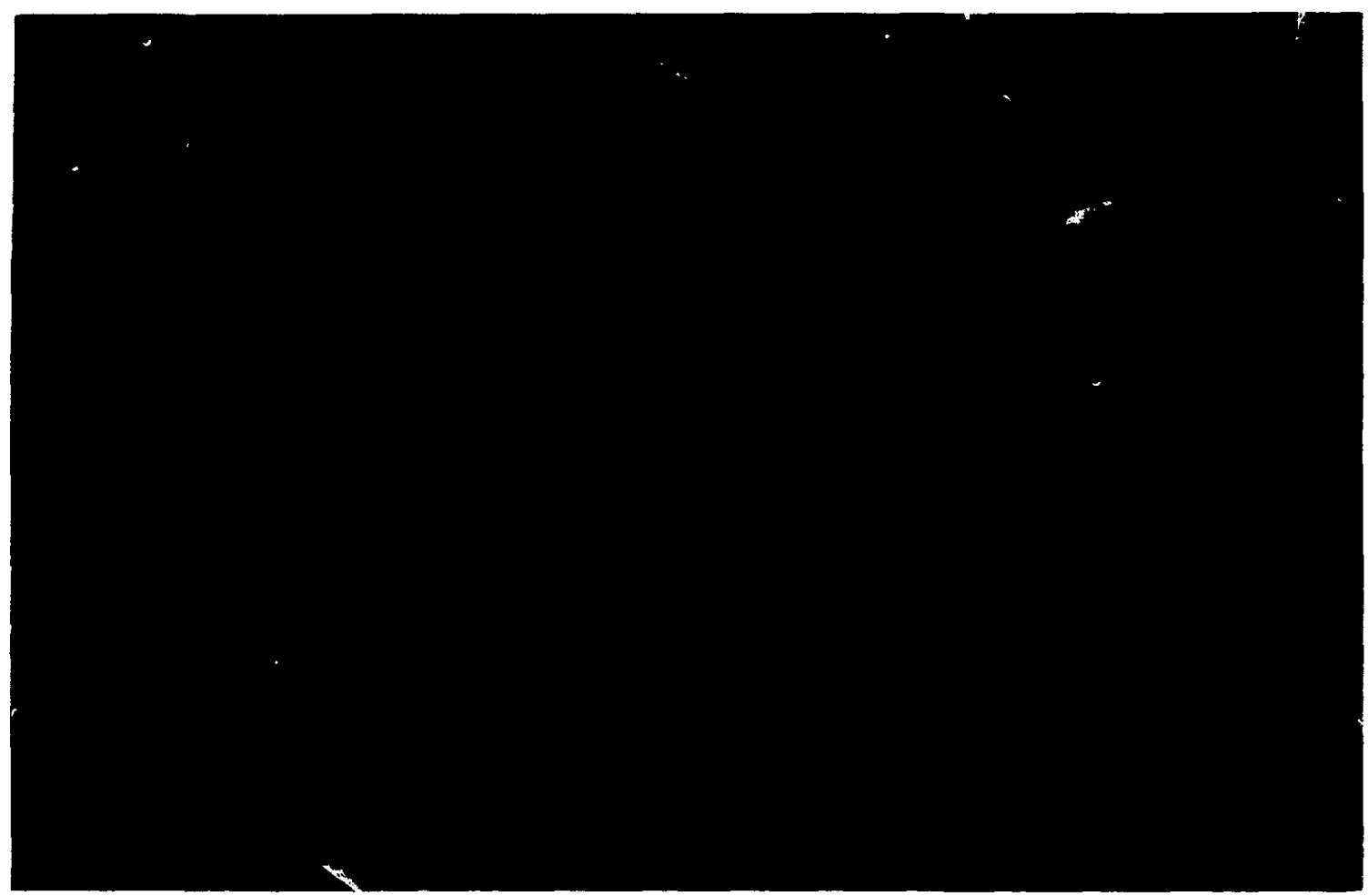

Fig. 69. Neutrino facility: proposed pion production target assembly (1982).

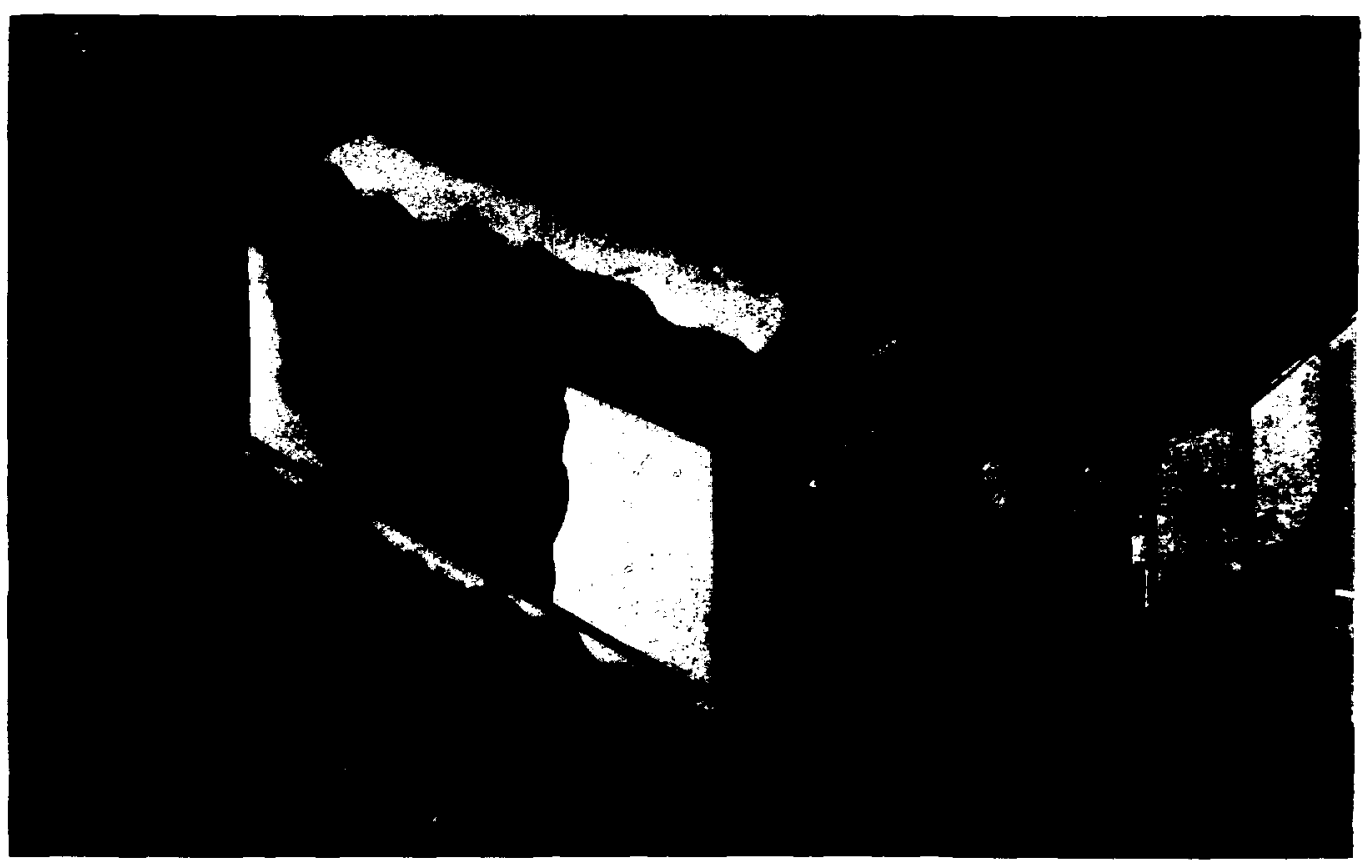

Fig. 70. Neutrino facility: proposed neutrino detector building (1982). 


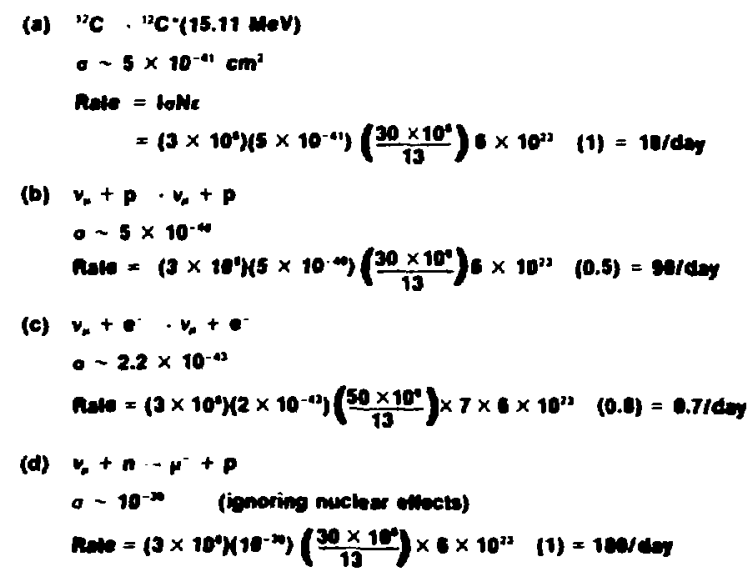

Fig. 71. Neutrino facility: estimated rates of neutrino interactions. 